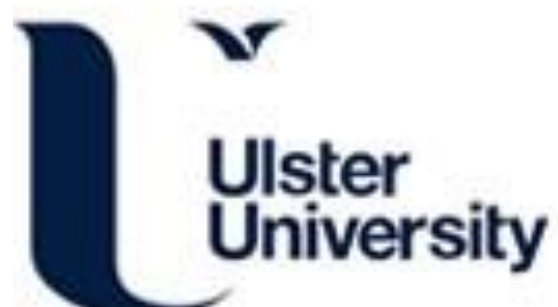

\section{A non-linear time-series analysis approach to identify thresholds in associations between population antibiotic use and rates of resistance}

THRESHOLDS study group (2019). A non-linear time-series analysis approach to identify thresholds in associations between population antibiotic use and rates of resistance. Nature Microbiology, 4(7), 1160-1172. https://doi.org/10.1038/s41564-019-0410-0

Link to publication record in Ulster University Research Portal

Published in:

Nature Microbiology

Publication Status:

Published (in print/issue): 01/07/2019

DOI:

10.1038/s41564-019-0410-0

\section{Document Version}

Author Accepted version

\section{General rights}

Copyright for the publications made accessible via Ulster University's Research Portal is retained by the author(s) and / or other copyright owners and it is a condition of accessing these publications that users recognise and abide by the legal requirements associated with these rights.

\section{Take down policy}

The Research Portal is Ulster University's institutional repository that provides access to Ulster's research outputs. Every effort has been made to ensure that content in the Research Portal does not infringe any person's rights, or applicable UK laws. If you discover content in the Research Portal that you believe breaches copyright or violates any law, please contact pure-support@ulster.ac.uk. 
1 A non-linear time-series analysis approach to identify thresholds in 2 associations between population antibiotic use and rates of resistance

3

4
José-María López-Lozano ${ }^{1}$, Timothy Lawes ${ }^{2 *}$, César Nebot $^{3}$, Arielle Beyaert ${ }^{4}$, Xavier Bertrand $^{5}$, Didier Hocquet ${ }^{5}$, Mamoon Aldeyab ${ }^{6}$, Michael Scott ${ }^{7}$, Geraldine Conlon-Bingham ${ }^{7}$, David Farren ${ }^{8}$, Gábor Kardos ${ }^{9}$, Adina Fésüs ${ }^{10}$, Jesús Rodríguez Baño ${ }^{11}$, Pilar Retamar ${ }^{11}$, Nieves Gonzalo-Jiménez ${ }^{12}$, Ian M Gould ${ }^{13}$, the THRESHOLDS study group ${ }^{14}$

${ }^{1}$ Medicine Preventive-Infection Control Team, Hospital Vega Baja, Orihuela-Alicante, Spain.

${ }^{2}$ The Wellcome Trust Liverpool-Glasgow Centre for Global Health Research, Liverpool, UK.

${ }^{3}$ Centro Universitario de la Defensa (CUD) de San Javier, Murcia, Spain.

${ }^{4}$ Departamento de Métodos Cuantitativos para la Economía y la Empresa, University of Murcia, Spain

${ }^{5}$ Centre hospitalier régional et universitaire (CHRU) and UMR 6249 Chrono-environnement, Université de Bourgogne-Franche-Comté, Besançon, France

${ }^{6}$ School of Pharmacy and Pharmaceutical Science, Ulster University, Northern Ireland, UK

${ }^{7}$ Pharmacy Department, Northern Health and Social Care Trust and Regional Medicines Optimisation Innovation Centre, Antrim, Northern Ireland, UK.

${ }^{8}$ Department of Medical Microbiology, Antrim Area Hospital, Antrim, Northern Ireland, UK

${ }^{9}$ Department of Medical Microbiology, Faculty of Medicine, University of Debrecen, Debrecen, Hungary

${ }^{10}$ Clinical Pharmacy, University of Debrecen, Debrecen, Hungary

${ }^{11}$ Infectious Diseases and Clinical Microbiology Unit, Instituto de Biomedicina de Sevilla (IBiS), Hospital Universitario Virgen Macarena and University of Seville, Seville, Spain.

${ }^{12}$ Medical Microbiology Department, Hospital Vega Baja, Orihuela-Alicante, Spain.

${ }^{13}$ Medical Microbiology Department, Aberdeen Royal Infirmary, Aberdeen, Scotland, UK.

${ }^{14} \mathrm{~A}$ full list of authors can be found at the end of the article

*Corresponding author:

E-mail: t.lawes@nhs.net (TL)

Short title: Population antibiotic use thresholds

Word count:

Abstract: 200

Text: 3157 (Introduction 478; Results 1863, Discussion 816)

Figs in manuscript: 5

Tables in manuscript 1

Extended data file including 3 figs, 6 tables 
Balancing access to antibiotics with control of antibiotic resistance is a global public health priority. Currently, antibiotic stewardship is informed by a 'use it and lose it' principle, in which population antibiotic use is linearly related to resistance rates. However, theoretical and mathematical models suggest use-resistance relationships are non-linear. One explanation is that resistance genes are commonly associated with 'fitness costs', impairing pathogen replication or transmissibility. Therefore, resistant genes and pathogens may only gain a survival advantage where antibiotic selection pressures exceed critical thresholds. These thresholds may provide quantitative targets for stewardship: optimising control of resistance while avoiding over-restriction of antibiotics. We evaluated the generalisability of a nonlinear time-series analysis approach for identifying thresholds using historical prescribing and microbiological data from five populations in Europe. We identified minimum thresholds in temporal relationships between use of selected antibiotics and rates of carbapenem-resistant Acinetobacter baumannii (in Hungary), extended spectrum $\beta$-lactamase producing Escherichia coli (Spain), cefepime-resistant Escherichia coli (Spain), gentamicin-resistant Pseudomonas aeruginosa (France), and methicillin-resistant Staphylococcus aureus (Northern Ireland) in different epidemiological phases. Using routinely generated data, our approach can identify context-specific quantitative targets for rationalising population antibiotic use and controlling resistance. Prospective intervention studies restricting antibiotic consumption are needed to validate thresholds. 


\section{Introduction}

100 Antimicrobials have facilitated improvements in health and food-security worldwide.

101 However, excessive use has promoted antimicrobial resistance (AMR), undermining many

102 aspects of healthcare and generating new disease burdens. ${ }^{1-4}$ While decisions around

103 antimicrobial use are made by individual prescribers and patients, consequences of over-use

104 are only fully apparent across populations. ${ }^{5}$ Competing needs and non-exclusivity lead to a

105 'tragedy of the commons'. ${ }^{1,2}$ The Global Action Plan on AMR therefore emphasises cross-

106 societal efforts to conserve the current stock of effective antimicrobials. ${ }^{6}$ Antimicrobial

107 stewardship aims to control AMR by reducing inappropriate use, while ensuring access to

108 effective therapy. ${ }^{2}$ There is growing evidence that reducing population use of antimicrobials

109 selecting for resistance, can effectively reduce AMR. ${ }^{7}$ However, over-restriction may be

110 counter-productive: use of alternative antimicrobials may increase in response, leading to

111 new selection pressures or resistance problems. ${ }^{8}$ Balancing the benefits and risks of

112 antimicrobials in healthcare requires understanding of their effects on AMR at population

113 scales. $^{9-11}$

114

Most empirical evidence has considered linear use-resistance relationships. ${ }^{12-16}$ Although useful for identifying the most salient selection pressures in a population, ${ }^{12}$ theoretical and mathematical models suggest non-linear relationships are more likely. ${ }^{9-11,17}$ In particular, selection pressures may only impact on resistance rates, where volumes of antibiotic use in the population are above minimum thresholds. Potential explanations for minimum thresholds, include: antibiotic substitution with changes in use ${ }^{8}$ associations between the strength of selection pressure and induction of co-resistance, ${ }^{18}$ differential effects on subpopulations within bacterial species, ${ }^{19}$ or ecological niches; ${ }^{20}$ and the 'total use thresholds' hypothesis. ${ }^{9}$ The latter concept arises from the observation that resistance genes are commonly associated with fitness costs impairing pathogen replication or transmissibility. ${ }^{21}$ Resistant pathogens may therefore only gain a survival advantage over susceptible organisms, where selection pressures from antibiotic use outweigh fitness costs. ${ }^{9}$ If identified, minimum thresholds may offer important targets for antibiotic stewardship, indicating an upper limit of safe population antibiotic use which does not increase resistance within populations.

Non-linear relationships between fluoroquinolone use and resistant E.coli and Staphylococcus aureus have been reported. ${ }^{20,22}$ We have shown that reducing antibiotic use to below minimum thresholds predicted shifts in the molecular and clinical epidemiology of S. aureus and Clostridium difficile. ${ }^{23-25}$ However, early findings suggest antibiotic use-resistance relationships are likely to depend upon the clinical population, ${ }^{9,23,25}$ pathogen genotypes, ${ }^{24}$ intensity of infection control, ${ }^{23}$ and transmission dynamics. ${ }^{11}$ Moreover, fitness costs associated with different resistance genes are variable. ${ }^{21}$ If fitness costs are low, or attenuated through epistasis ${ }^{26}$ or compensatory mutations, ${ }^{27}$ resistance may persist despite removing previously important antibiotic selection pressures. ${ }^{28}$ A generalisable method for finding minimum thresholds should therefore provide context-specific results, have relatively lowinformational demands, and allow for iteration.

Here, we examined the generalisability of a non-linear time-series analysis methodology for identifying minimum threshold in use-resistance associations. Using routinely generated data from five European centres, we show this pragmatic approach can provide populationspecific quantitative targets for antimicrobial stewardship for a variety of resistance outcomes, epidemiological phases, transmission dynamics, and clinical populations. 
148

149

150

151

152

153

154

155

156

157

158

159

160

161

162

163

164

165

166

167

168

169

170

171

172

173

174

175

176

177

178

179

180

181

182

183

184

185

186

187

188

189

190

191

192

193

194

195

196

197

\section{Results}

\section{Identifying non-linear temporal relationships: from experiment to application}

In a Monte Carlo experiment we compared the ability of linear and non-linear time-series analysis (Multivariate Adaptive Regression Splines, MARS) to identify pre-defined relationships between simulated explanatory and outcome time-series (Supplementary Figure 1). Non-linear time-series analysis (NL-TSA) accurately identified both truly linear and nonlinear associations. However, linear time-series analysis provided biased estimations and overall poorer data-fit if relationships were non-linear.

NL-TSA models applied to retrospective time-series data from five European study populations (examples 1-5), frequently identified minimum thresholds in antibiotic useresistance relationships, (figures 1-5 and Supplementary Table 1). 'Ceiling effects', in which further increases in explanatory variables did not affect resistance rates, were found at highlevels of use of some antibiotics and hand hygiene. Non-linearities in autoregression and population interaction terms further indicated the complexity of transmission dynamics within and between clinical populations.

\section{Example 1: Carbapenem-resistant Acinetobacter baumannii (Debrecen, Hungary)}

We examined ecological determinants of carbapenem-resistant A. baumannii (CRAb) in a tertiary hospital population in Debrecen, Hungary (figure 1). Between Oct 2004 and Aug 2016 ( $\mathrm{n}=143$ months), incidence density of CRAb increased from a 12-month average of 0.14 to 9.43 cases per 10000 OBDs, while that of carbapenem-susceptible A. baumannii (CSAb) remained relatively constant. There were no planned antibiotic stewardship interventions in the study period. We observed increasing use of broad-spectrum antibiotics, including a tripling in carbapenem use, and more recent escalation in colistin use.

Carbapenem resistance in this setting was conferred predominantly by bla $a_{\text {OXA-23-like }}$ carbapenemases, while bla $a_{\text {OXA-24-like }}$ genes occurred sporadically. ${ }^{29} \mathrm{CRAb}$ were significantly more likely to be resistant to ciprofloxacin, gentamicin, amikacin, piperacillin-tazobactam and ceftazidime than CSAb (Supplementary Table 2), although susceptibility testing for the latter two agents was discontinued in 2013 as recommended by EUCAST. Vector autoregression models found that colistin use followed, rather than predicted, variation in $\mathrm{CRAb}$ incidence density. Use of carbapenems, fluoroquinolones, piperacillin-tazobactam, third generation cephalosporins (3GC), and aminoglycosides were considered potential explanatory variables.

Previous CRAb incidence density and recent hospital use of carbapenems, piperacillintazobactam, and fluoroquinolones were explanatory variables in the best-fit $\left(\mathrm{R}^{2}=0.86\right)$ nonlinear TSA model (Supplementary Table 1). In an almost identical model with poorer tradeoff of data fit and model complexity (higher Modified Generalised Cross Validation statistic), fluoroquinolone use was replaced by the effect of $3 \mathrm{GC}$ use above a threshold of $36(95 \% \mathrm{CI}$, 30 to 41 ) DDDs per 1000 OBDs (coefficient, $95 \%$ CI: $0.111,0.018$ to 0.203 ; $p=0.019$; lag 3 ).

In this setting increases in CRAb added to, rather than replaced, CSAb, suggesting CRAb occupied new ecological niches. Strong autoregression in the CRAb time-series was consistent with substantial within-hospital transmission. ${ }^{29}$ CRAb incidence density increased when population use of carbapenems, piperacillin-tazobactam, fluoroquinolones, and 3GC exceeded minimum thresholds. By the end of the study period, use of fluoroquinolones had reduced to below threshold. However, CRAb could be further controlled by reducing use of 
198 carbapenems, 3GC, and piperacillin-tazobactam, from present levels to respective thresholds

199 (table 1).

$200 \quad$ Example 2: Extended spectrum $\beta$-lactamase producing E. coli (Orihuela, Spain)

201 We examined variables temporal associated with the percentage of $E$. coli producing ESBL

202 (\%Ec-ESBL+) in connected district general hospital and community populations of Orihuela,

203 Spain, between Jul 1991 and Oct 2016 (n=304 months, figure 2). Limited outbreaks of

204 ESBL-producing E. coli were noted from 1998, but from 2002 the \%Ec-ESBL+ increased

205 rapidly alongside escalating use of fluoroquinolones and co-amoxiclav. While use of these

206 agents later stabilised or declined, hospital use of third-generation cephalosporins continued

207 to increase.

208

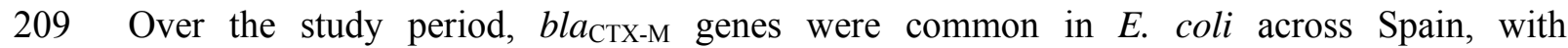
210 dissemination of the CTX-M-15-producing O25b-ST131 clone and clonally unrelated CTX211 M-14-producing strains identified. ${ }^{30,31}$. Acquisition of fluoroquinolone resistance was a key 212 step in the evolution of dominant bla ${ }_{C T X-M-15}$ containing sub-clones of O25b-ST131. ${ }^{32}$ 213 Consistent with this, 81\% of ESBL-producing E. coli in Orihuela were non-susceptible to 214 ciprofloxacin. They were also significantly more likely to be resistant to co-trimoxazole, coamoxiclav and aminoglycosides compared to non-ESBL E. coli (Supplemental Table 3). Vector autoregression models demonstrated bidirectional interactions between community and hospital \%Ec-ESBL+ and that use of piperacillin-tazobactam and carbapenems followed, rather than predicted, changes in \%Ec-ESBL+. In separate hospital and community models, $\%$ Ec-ESBL in the other population was considered as an explanatory variable.

In the best-fit model $\left(\mathrm{R}^{2}=0.62\right)$ hospital $\%$ Ec-ESBL+ was predicted by prior $\%$ EcESBL in hospital and community, and hospital use of $3 \mathrm{GC}$, and fluoroquinolones exceeding minimum thresholds (Supplementary Table 1). A potential 'ceiling' effect was noted at high-levels of fluoroquinolone use, meaning that when use exceeded a second upper threshold, further increases in \%Ec-ESBL+ were small. An initial decrease in \%Ec-ESBL+ where fluoroquinolone use was between 151 and 161 DDDs per 1000 OBDs reflected uncertainty around this ceiling threshold, which may be resolvable with additional data. In the model for community \%Ec-ESBL+ $\left(\mathrm{R}^{2} 0.767\right)$, associations were identified with hospital \%Ec-ESBL+, and community use of fluoroquinolones and co-amoxiclav above minimum thresholds.

Autoregressive and population interaction effects suggested the importance of horizontal transmission of ESBLs, with predominant influence of community on hospital epidemiology. Population use of broad-spectrum beta-lactams and fluoroquinolones were important explanatory variables in both settings. In the community, use of fluoroquinolones had fallen below threshold levels and \%Ec-ESBL+ had started to decrease by the end of the study. However, translating thresholds into antibiotic stewardship targets (table 1) suggested further restricting community co-amoxiclav use by $31 \%$ and hospital use of fluoroquinolones $(-41 \%)$ and $3 \mathrm{GC}(-21 \%)$.

From best-fit models, we created 24-month projections for \%Ec-ESBL+ in the hospital and community under different antibiotic stewardship options, and compared these to expected $\%$ Ec-ESBL+ under a 'business as usual' scenario of antibiotic use (Supplementary Figure 2). Immediate restriction of hospital use of fluoroquinolones and $3 \mathrm{GCs}$ to thresholds was predicted to cause an abrupt and sustained reduction in hospital \%Ec-ESBL+ from $9.89 \%$ to $2.35 \%(\mathrm{p}<0.0001)$ and, due to population interactions, a gradual reduction in community $\%$ Ec-ESBL+ from $7.11 \%$ to $3.69 \%(\mathrm{p}<0.0001)$. Limiting community co-amoxiclav use to 
threshold levels was predicted to cause a small reduction in community $\%$ Ec-ESBL+ but not to affect hospital epidemiology.

\section{Example 3: Cefepime-resistant Escherichia coli (Seville, Spain)}

We examined ecological variables explaining cefepime resistance among urinary or invasive E.coli infections (\%Ec-FepR) in a tertiary hospital in Seville, Spain. Between March 2008 and December 2016 ( $\mathrm{n}=108$ months) $\%$ Ec-FepR fell from $12.6 \%$ to $7.9 \%$ (figure 3). Cefepime use was low, declining from 4.4 to 1.6 DDDs per 1000 OBDs with thrice-weekly (Jan 2012, audit1) and daily (Jan 2014, audit2) prescription audits. By contrast, previously declining use of $3 \mathrm{GC}$ increased from January 2013 when they replaced co-amoxiclav and ciprofloxacin as first-line empirical therapy for intra-abdominal or urinary infections.

Resistance to cefepime in E. coli is mostly conferred by ESBLs with high affinity for cefepime (TEM-, SHV- and CTX-M-types). In addition to those agents hypothesised to predict ESBL-producing E. coli (see example 2), we considered the role of piperacillintazobactam. ${ }^{13}$ Due to low rates of cefepime prescribing, we grouped this with 3 GC use. We introduced variables for antibiotic auditing interventions and for revised susceptibility breakpoints (Oct 2014). ${ }^{33}$

The final non-linear model $\left(\mathrm{R}^{2}\right.$ 0.30) identified associations with \%Ec-FepR 12 months prior (seasonal effect) and use of third- or fourth-generation cephalosporins and fluoroquinolones above minimum thresholds (Supplementary Table 1). A significant interaction term between $\%$ Ec-FepR in the previous month (autoregression, lag 1) and the second antibiotic auditing intervention suggested a gradual effect of the audit in reducing $\% \mathrm{Ec}-\mathrm{FepR}$.

Reductions in third- and fourth-generation cephalosporins and fluoroquinolone use to below minimum thresholds explained modest declines in \%Ec-FepR between 2008 and 2012. Partial reversal in this trend was consistent with increasing use of $3 \mathrm{GC}$ towards the end of the study period. \%Ec-FepR could be controlled further by reducing third- and fourth-generation cephalosporin use by $41 \%$ (table 1 ).

\section{Example 4: Gentamicin-resistant Pseudomonas aeruginosa (Besançon, France)}

We examined ecological variables explaining rates of gentamicin-resistant $P$. aeruginosa (GRPa) among adult and paediatric admissions to a tertiary hospital in Besançon, France (figure 4). Between Jan 1999 and Dec 2014 (n=192 months), incidence density of GRPa decreased from 14.0 to 3.4 cases per $1000 \mathrm{OBDs}$, and the proportion of $P$. aeruginosa isolates resistant to gentamicin declined from $63 \%$ to $16 \%$.

Aminoglycoside modifying enzymes (AMEs) are the most common mediators of aminoglycoside resistance in $P$. aeruginosa; with acetyltransferases (e.g. $a a c\left(6^{\prime}\right)-I b$ ) and nucleotidyltransferases (e.g ant(2")-Ia) most frequent in Europe. ${ }^{34}$ Since related genes in mobile genetic elements encode AMEs and $\beta$-lactamases, $\beta$-lactam use may also predict aminoglycoside resistance. ${ }^{35}$ In previous analyses from Besançon, aminoglycosides, cefepime and fluoroquinolones were predictors of MexXY-OprM overproduction in P. aeruginosa. ${ }^{36}$ $\mathrm{GRPa}$ isolates were also more likely to overproduce the chromosomally-encoded AmpC cephalosporinase $(56 \%$ vs. $20 \% ; \mathrm{p}<0.001)$ and be multi-drug resistant $(65 \%$ vs. $13 \%$; $\mathrm{p}<0.001)$. We hypothesized that GRPa incidence density may be predicted by use of aminoglycosides, fluoroquinolones, extended-spectrum penicillins with $\beta$-lactamase inhibitors, carbapenems, monobactams, and third- and fourth-generation cephalosporins. 
Given potential intra-class differences in promoting resistance, we grouped use of gentamicin

In the best-fit model $\left(\mathrm{R}^{2}=0.86\right)$, GRPa incidence density was strongly predicted by incidence density in the previous month, and hospital use of gentamicin/tobramycin and fluoroquinolones above minimum thresholds (Supplementary Table 1). No independent association with Amikacin use was identified.

Declining GRPa was largely explained by falling inpatient use of gentamicin/tobramycin, and, to a lesser extent, fluoroquinolones. Use of both drug groups was maintained below minimum thresholds from around 2007. Continuing decreases in GRPa incidence density were at least partially explained by autocorrelation at lower incidence densities. Reciprocal increases in gentamicin-susceptible $P$. aeruginosa over the same period, and moderate inverse correlation $(\mathrm{r}=-0.55)$, suggest competition for the same niche as GRPa. Gentamicin/tobramycin and fluoroquinolone use should be maintained below thresholds to control GRPa.

\section{Example 5: Methicillin-resistant Staphylococcus aureus (Antrim, Northern Ireland) \\ We evaluated ecological variables explaining incidence density of MRSA clinical isolates in adult admissions to a district general hospital in Antrim (Jan 2005 to Sep 2013, n=105 months). Between 2005 and mid-2008, incidence density of MRSA clinical isolates remained stable at c.3.0 per 1000 OBDs (figure 5). Following restrictiong fluoroquinolones (January 2008) and intensification of hand-hygiene, burdens fell to 1.64 cases per 1000 OBDs by 2013.}

The epidemic hospital MRSA clonal complex CC22, predominated in Northern Ireland during the study period: its success attributed to an ability to acquire mobile genetic elements carrying multiple resistance genes, with limited fitness costs. ${ }^{37}$ Following prior linear timeseries analyses from the region, ${ }^{14}$ we hypothesised use of fluoroquinolones, 3GC, coamoxiclav, and macrolides could be important predictors of MRSA epidemiology.

In the best-fit model $\left(\mathrm{R}^{2}=0.53\right)$, MRSA incidence density was positively associated with rates of MRSA in the previous month and use of fluoroquinolones, 3GC, and co-amoxiclav exceeding minimum thresholds (Supplementary Table 1). An inverse relationship was seen with increased hospital use of alcohol-based hand rub (ABHR) up to 6.9 Litres per 1000 OBDs, above which further increases in ABHR use was not associated with further declines in MRSA.

Declining MRSA incidence density was partly explained by deliberate restriction of fluoroquinolone use, and concurrent declines in co-amoxiclav and 3GC. Strong autoregression, and inverse association with ABHR use, were consistent with importance of infection control measures in interrupting horizontal transmission. Reversal of previous declines in fluoroquinolone use were seen by the last year of study. Findings suggested maintaining use of co-amoxiclav and 3GC under thresholds, use of ABHR at threshold levels, and further restriction of fluoroquinolones (table 1). 


\section{Discussion}

347 Using a non-linear time-series analysis (NL-TSA) approach, we found empirical evidence of

348 non-linear relationships between population antibiotic use and resistance rates in five

349 European settings. The method was generalisable to different clinical populations, resistant that identification of minimum thresholds, and associated confidence intervals, could provide population-specific quantitative targets for antibiotic stewardship.

Our approach builds upon earlier work using linear time-series analysis to explain temporal relationships between antibiotic use and resistance. ${ }^{12-16}$ NL-TSA shares a number of strengths, including: low-informational demands; ease of reiteration as new data becomes available; adjustment for the non-independence of serial observations and stochasticity inherent to time-series of communicable diseases; identification of temporality in associations and 'lagged' effects; and integration of impacts of multiple exposures. ${ }^{12}$ Additionally, NLTSA reveals non-linear relationships, providing more accurate understanding of how modifying antibiotic use, infection control or other exposures is likely to affect resistance. Limitations of NL-TSA include: the need for longer time-series than linear TSA; the potential for spurious thresholds in areas of limited data or extremes of the exposure variable range; and difficulty in identifying thresholds in situations of stable resistance and prescribing. We note the poorer predictive performance of some models (e.g. example 3), may be explained by absence of data on infection prevention and control activities, and resistance levels in interacting populations.

Our findings may have important implications for antibiotic stewardship. In general, impacts of changes in antibiotic use on resistance vary dependent upon the antibiotic use level. More specifically, minimum thresholds may be interpreted as an upper limit for 'safe' population antibiotic use which does not appear to substantially increase resistance rates at the population level. Alternative theories may suggest the threshold indicates: a maximum level of selection pressure not conferring a survival advantage to resistant pathogens to spread within populations or ecological niches, ${ }^{8,12}$ or strong enough to induce resistance, ${ }^{18}$ or a minimum level of use, below which antibiotic substitution creates equivalent or greater selection pressure. ${ }^{8}$ Crucially, they may provide quantitative targets for balancing the need to access therapies with control of resistance, analogous to 'quotas' applied to other natural resources which seek to maximize extracted value while maintain a non-declining stock. ${ }^{1,2,38}$ Moving from qualitative targets of reducing use, to quantitative targets may also aid operational effectiveness. Targets appear to work best if pragmatic, collaborative and iterative. ${ }^{39}$ Complete restriction of use of agents is rarely feasible: in balancing access to effective therapies with control of resistance, quantitative targets could align interests of clinicians and antimicrobial management team. ${ }^{40}$

We emphasise the need for caution with interpretations of thresholds. Firstly, thresholds should offer guidance rather than strict limits. Uncertainty around thresholds is variable, as reflected in width of associated confidence intervals. Narrower confidence intervals around threshold locate with reasonable precision the level of antibiotic use at which effects on resistance are substantially altered. Wider intervals may indicate insufficient data, or the influence of additional explanatory variables. In rare instances of multiple closely occurring thresholds in a single functional relationship, the width of confidence intervals may be underestimated and should be interpreted with particular caution. We suggest a pragmatic approach, of interpreting thresholds depending upon the policy scenario. Where the priority is strict control of resistance a conservative approach of limiting use to the lower limit of the 
threshold confidence interval is advisable. Where excessive restriction is a concern, the standard approach of limiting use to the point estimate of the threshold is likely to offer the best balance between restriction and control of resistance. Secondly, changes in molecular epidemiology under sustained antibiotic selection pressures, such as compensatory mutations minimising fitness costs, ${ }^{26,27}$ or strain replacement, ${ }^{24,25}$ may mean thresholds vary by epidemiological phase and time. Variation in thresholds across populations can be anticipated, reflecting host, environment, and organism factors. ${ }^{23-25}$ Therefore, models based on local data and iterative analysis is necessary to ensure time and context-specific guidance. Thirdly, it is important not to assume that all antibiotic use below thresholds is safe, since antibiotic exposures may be important for individual patients, or cause unseen change in reservoirs of resistant pathogens in environment or hosts.

Potential foci for further research include: evaluating the consistency of thresholds for specific antibiotic use-resistance combinations across different settings and identifying factors affecting thresholds; applying NL-TSA to composite indices of resistance; ${ }^{41,42}$ use of Bayesian approaches in selection of explanatory variables and analysis with short time-series or rare resistance outcomes; ${ }^{43}$ and prospective studies to validate the effectiveness of quantitative targets in antibiotic stewardship.

We have illustrated how non-parametric time-series models based on empirical data can identify non-linear relationships between population antibiotic use and resistance burdens. Further we have shown how identification of population-specific minimum thresholds may guide rational compromises between control of resistance and access to therapeutics. With the increasing availability of electronic surveillance and healthcare systems, this approach offers a useful tool for sustaining the effectiveness of current antimicrobials in many areas of the world. 


\section{Design and study populations}

This was a multi-centre time-series study. We used multivariable non-linear time-series analysis to quantify associations between ecological exposures, including population use of antibiotic groups, and rates of antibiotic-resistant infections in five populations from France, Hungary, Northern Ireland (UK), and Spain (Supplementary Table 5).

The populations and resistance outcomes were a purposive sample, chosen to reflect varying epidemiological scenarios, clinical settings, and resistant infections in European centres participating in the THRESHOLDS (THReshold EStimation to Help Optimise Local Decisions on antibiotic Stewardship) study group. This collaborative aims to further the development of time-series analysis for understanding antibiotic resistance and planning antibiotic stewardship. Members included centres with prior experience in applying linear time-series approaches. Investigators were asked to identify an important resistance problem in a defined clinical population from their region. Minimum data requirements were consistent microbiological and prescribing data across a minimum of 60 monthly observations ( 5 years). Duration of time-series was defined by the longest period of consistent data for a minimum data set of the outcome and candidate explanatory variables.

For each population we described the regional scenario for the chosen outcomes, the theoretical basis for inclusion of candidate explanatory variables, findings from the non-linear time-series analysis, and how these could inform local antibiotic stewardship policy.

\section{Outcome and explanatory time series}

The outcome time-series for each population were: carbapenem-resistant Acinetobacter baumannii (Debrecen, Hungary); extended spectrum $\beta$-lactamase producing Escherichia coli in hospital and community (Orihuela, Spain); cefepime-resistant Escherichia coli (Seville, Spain); gentamicin-resistant Pseudomonas aeruginosa (Besançon, France); and methicillinresistant Staphylococcus aureus - MRSA (Antrim, Northern Ireland). Cases were defined microbiologically as isolates from all relevant body sites not identified as infection control specimens and meeting consistent criteria for resistance or resistance mechanism (see supplemental file for details). Isolates from the same patient identified within 30 days of a prior isolate with the same organism were considered part of the same infectious episode and de-duplicated. Outcomes were expressed, where possible, as monthly incidence density of resistant infections (cases per 1000 or 10,000 occupied bed days, OBDs). Where there were large changes in testing frequency or organism identification over time, we defined resistance as a percentage of clinical isolates from the same organism with any susceptibility pattern.

The primary explanatory variables were monthly population use of antibiotic agents classified by pharmacological sub-group of antibacterials for systemic use (J01) in the 2016 WHO/ATC index, and expressed as defined daily doses (DDDs) per 1000 OBDs (hospital) or 1000 inhabitant-days (community). Candidate antibiotic sub-groups were identified a priori, on the basis of regional co-resistance profiles, molecular epidemiology in the region, reviews and prior evidence on individual or population level risk factors for acquisition of the resistant infection. We included separate time-series for individual antibiotic agents or chemical sub-groups only where there were strong theoretical grounds for investigating independent associations, such as prior evidence of variable within-class actions or targeting within antibiotic stewardship interventions. 
496 In addition, we incorporated autoregressive terms capturing association between current 497 incidence density and incidence density in recent months. Where available, we incorporated 498 infection prevention and control (IPC) variables such as use of alcohol-based hand-rub. 499 Dummy variables were added to capture immediate and gradual impacts of changes in 500 laboratory methods or other planned interventions. We considered lags in association of up to 5016 months and seasonal autoregressive terms (lag 12).

502

510 Pathogens were identified using standard laboratory methods. Susceptibility testing was by 511 disc-diffusion (Besançon, Debrecen, Antrim) or broth microdilution (Seville, Orihuela). 512 Isolates were defined as resistant if not susceptible to an antibiotic agent according to zone 513 diameter (for disc-diffusion) or minimum inhibitory concentration (MIC, for broth 514 microdilution) breakpoints as recommended by the European Committee on Antimicrobial 515 Susceptibility Testing (EUCAST) or Clinical \& Laboratory Standards Institute (CLSI). 516 Details of standards used, and deviations from EUCAST or CLSI criteria are detailed in table

517 i. Known changes in breakpoints and laboratory methods were adjusted for in time-series 518 analysis.

\section{Statistical methods}

In the following sections we provide a technical exposition of the statistical methods used. We offer here, a brief description for the general reader.

Before applying non-linear time-series analysis (NL-TSA) to real-world datasets from study populations, we performed a statistical, Monte-Carlo, experiment to illustrate its advantages over, more familiar, linear TSA. We applied both linear and non-linear TSA to computersimulated time-series, where the relationship between the outcome and explanatory timeseries was known. Three types of relationship were explored: a linear relationship; a nonlinear relationship without correlations between successive data points in time-series (autoregression), and a non-linear relationship with autoregression; By running this experiment over 10,000 simulated datasets for each type of relationship we evaluated the typical ability of linear and non-linear TSA to describe the relationships accurately.

We next applied a seven-step non-linear time-series analysis approach to resistance problems in study populations (figure 6).

Mirroring more familiar regression techniques, we started by defining a set of explanatory variables including antibiotic use, infection control, population interactions, alongside terms for autoregression. This set was defined by (a) expert opinion informed by prior risk-factor studies, molecular epidemiology in the region; and (b) inspection of resistance profiles of the pathogen of interest. We consider delays between changes in explanatory time-series and associated change in outcome time-series (lags) of up to 6 months. Before analysis we checked time-series and make adjustments for extreme values (outliers) or unexpected shifts in mean (structural changes). We also used vector autoregression (VAR) models to help distinguish any reverse causality in relationships between explanatory and outcome series: 
this might occur, for example, if prescribing behaviour was altered by resistance rates in the population in previous months. Next we restrict the set of explanatory variables, and lags, to be put into final multivariable models. We use a procedure that fits smooth functions to relationships between explanatory and outcome time-series, and allows visual inspection of likely significant associations. After identifying the most promising explanatory variables (and lags) we enter these into a Multivariate Adaptive Regression Splines (MARS) model which both identifies significant predictors, and defines any non-linear relations as a series of linear relationships connected by 'knots' or thresholds. Model fit for MARS is checked by ensuring residuals were normally distributed without unexplained non-linearities. Confidence intervals around each threshold were fit by a conditional conservative inverted likelihood ratio (CCILR) method, using partial residuals. Finally, we converted thresholds from models into suggested maximum total treatment courses per month in the population by multiplying model thresholds by the size of the population and dividing by an average treatment course.

\section{Figure 6: Summary of the 7-step non-linear time-series methodology and potential pitfalls}

Step 1: Theoretical foundation

Identification of antibiotic sub-groups or agents, infection control interventions, and other

ecological variables hypothesised to predict the outcome:

- Prior evidence on risk-factors

- Regional molecular epidemiology

- Most frequent co-resistances in antibiograms of resistant pathogen

Step 2: Data validation

Review of time-series to identifiy unanticipated step-wise changes in time-series (Chow's test), or outliers (Cooks distances) and to make appropriate adjustment or replacements

Step 3: Identifying the most likely predictors and their lags

- Direction of temporal association explored by vector autoregression (VAR) analyses

- Significant associations (and lags) identified by inspection of plots from multivariable General Additive Model (GAM) plots.

Step 4. Multivariable model estimation

- Multivariate Adaptive Regression Splines (MARS) procedure to select and estimated final multivariable models, and describe associations in terms of one or more stable linear functions linked by thresholds or 'knots'.

Step 5: Diagnostic checks of model fit

- Normal distribution of residuals with mean zero, and homogenous variance

- Absence of residual autocorrelation, evaluated by autocorrelation function (ACF) plots

- Absence of residual non-linearities in Hinich Test

Step 6. Confidence intervals for thresholds

- Conditional conservative inverted Likelihood Ratio (CCILR) confidence intervals for each threshold generated using partial residuals after adjustment for other covariates and thresholds.

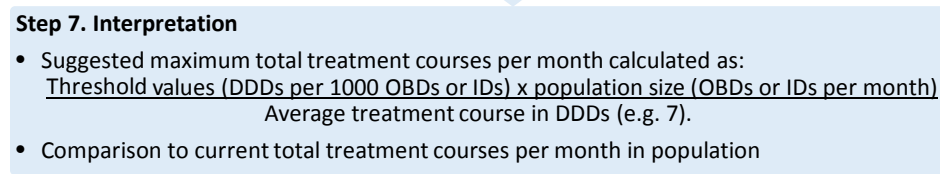


564

To provide an example of how model findings can inform policy, for the Orihuela population, we predicted the effects of restricting antibiotic use to threshold levels compared to a 'business as usual' scenario of prescribing based on the last months of study. We quantify impacts of immediate restriction sustained over two years.

A technical explanation follows:

\section{a) Comparing linear and non-linear time-series model performance}

We used a Monte-Carlo experiment to compare the ability of linear (Ordinary Least Squares, OLS) and non-linear (Multivariate Adaptive Regression Splines, MARS) time-series models in identifying various pre-defined functional relationships between simulated explanatory and outcome time-series. We hypothesised that for time-series related by simple linear processes MARS and OLS regression methods would perform equally well, but that only MARS would accurately identify non-linear associations. We generated 10,000 simulated datasets using simple stochastic processes incorporating the following pre-defined functional relationships:

(i) Non-autoregressive without threshold

$$
y_{t}=-4+2 x_{t}+u_{t} \quad u_{t} \sim N\left(0, \sigma^{2}=0.02\right) \quad \forall t=1, \cdots, 200
$$

(ii) Non-autoregressive with threshold

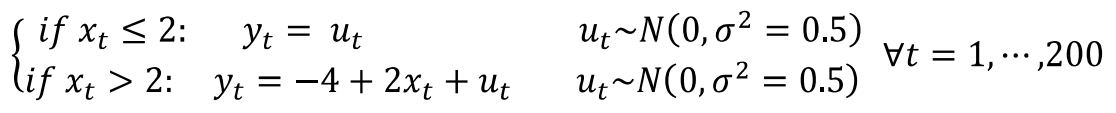

(iii) Autoregressive with threshold

$$
\left\{\begin{array}{ccc}
\text { if } x_{t} \leq 0: & y_{t}=0+\rho y_{t-1}+u_{t} & u_{t} \sim N\left(0, \sigma^{2}=0.05\right) \\
\text { if } x_{t}>0: & y_{t}=0+\rho y_{t-1}+1 x_{t}+u_{t} & u_{t} \sim N\left(0, \sigma^{2}=0.05\right)
\end{array} \forall t=1, \cdots, 200\right.
$$

Where:

$x_{t} \quad$ is the explanatory (independent) time-series variable at time $t$

$y_{t} \quad$ is the outcome (dependent) time-series variable at time $t$

$u_{t} \quad$ is the error term at time $t$, with Normal distribution, zero mean and variance $\sigma^{2}$

$\rho y_{t-1} \quad$ is an autoregressive term or order 1 (i.e. dated at $t-1$ ) with $\rho=0.25$

For each dataset we fitted both linear and non-linear time-series analyses, and recorded sample parameter estimates ( $a$ constant, $b$ slope, and $s^{2}$ as the estimate of population variance) and a measure of goodness of fit $\left(\mathrm{R}^{2}\right)$. Histograms were created illustrating the distributions of $\mathrm{R}^{2}$ values and parameter estimates from both linear and non-linear models. Visual comparison was made to pre-defined parameter values to identify bias in parameter estimates. We used a t-test of mean difference for independent samples to compare model performance based on $\mathrm{R}^{2}$ values.

\section{b) Applications of non-linear time-series analysis to real-world datasets}

We applied a seven-step approach to generate non-linear time-series models describing how contemporaneous and prior population antibiotic use, and other ecological variables, explain monthly variation in clinical burdens from antibiotic-resistant infections in five European centres. 
614 Participating centres identified a priori a minimum dataset of antibiotic sub-groups or agents

615 they considered most likely to affect the epidemiology of the resistant organism under

616 investigation (target organism). Decisions were based upon: previous empirical evidence of

617 risk factors and molecular epidemiology in the study region or related contexts. Additionally,

618 using antibiogram data from the study period and population, we reviewed co-resistances to

619 other antibiotics among isolates of the target organism with and without the resistance under

620 investigation. We considered antibiotics with the largest absolute rates of co-resistance in the

621 resistant isolates to be most likely to exert significant selection pressures. ${ }^{44,45}$ Consensus on

622 the list of potential predictive variables was found through discussion among all

623 THRESHOLDS study group members.

624 Where data was available we integrated additional explanatory variables on hospital activity

625 or infection control activities, associated with the outcome variable in previous studies.

\section{Step 2: Data validation}

To ensure consistent time-series we first accounted for known changes in exogenous conditions, such as changes in laboratory method. We captured immediate and gradual effects by entering a dummy variable ( 0 in months before the change, 1 in months after change) and its interaction with an autoregressive term, as explanatory variables. We then reviewed timeseries to detect possible unknown measurement errors as follows. Visual inspection identified potential structural changes (seen as large step-wise change in mean for instance) or outliers (seen as values deviating substantially from surrounding values). Successive Chow tests were applied to automatically detect the most probable dates of structural changes in the timeseries and, where necessary, to disaggregate the sample into two or more segments, each with a stable mean. For each segment we applied an outlier detection technique using the following criterion: an observation was considered as an outlier if Cook's distance at this point was greater than five times the mean of Cook's distances of all the observations of the segment. Finally, we replaced outlier values with the mean of the three preceding and three following observations.

\section{Step 3 Identifying the most likely predictors and their lags}

Given the potentially complex relationships between ecological variables under investigation we sought to refine our understanding of potential associations before applying final multivariable non-linear models.

Firstly, situations of reverse causality could exist when ecological exposures - such as rates of infections with resistant pathogens connected populations, or use of some antibiotic groups in a given population - respond to, rather than predict, rates of resistance. In order to minimise this risk, we tested direction of temporal relationships between explanatory and outcome time-series by applying Granger-causality analysis and Vector Autoregression (VAR) models. Secondly, non-linear models of the type used in this study are potentially complex and difficult to extract form the data if too many predictors are used at the same time. Therefore, we carried out an additional a priori data-based selection of candidate explanatory variables and lags (the lag refers to the delay in months between change in exposure and associated change in outcome). This was done through inspection of outputs from fitting a General Additive Model (GAM) to the data. GAM is a very general procedure that can be 
701

702

703

used for the identification of the most likely predictors, since it runs a non-parametric estimation of the functional relationships between explanatory $(x)$ and outcome $(y)$ timeseries, based upon iterative data fitting, rather than prior assumptions. It also allows for variability in the functional relationships across different values of the explanatory variables and can therefore capture non-linear associations between ecological variables and resistance outcomes. ${ }^{49}$ In particular, we used the GAM procedure in the SCAB34S Splines module (available in SCA Workbench, Scientific Computing Associates Corp, Illinois, USA) to define the relationship between $p$ explanatory $(x)$ and the outcome $(y)$ time-series as a sum of smooth, or spline, functions:

$E\left(y \mid x_{1}, x_{2}, \ldots, x_{p}\right)=s_{0}+\sum_{j=1}^{p} s_{j}\left(x_{j}\right)$

where $\left(s_{j}\left(x_{j}\right)\right)$ are the spline functions; they were standardised such that, after removal of free constants $\left(s_{o}\right)$ their expected contribution to the outcome $(y)$ is zero (i.e. $\operatorname{Es}_{j}\left(x_{j}\right)=0$ for each $j$ ).

The splines were derived by a process of splitting the time-series into sections, joined at knot points, and fitting simple curves described by cubic functions to the data in each section. The GAM methodology identified the optimal combination of spline functions $s_{j}\left(x_{j}\right)$, following the iterative procedure suggested by Hastie and Tibshirani(1986). ${ }^{46}$ Combining a local scoring algorithm and a backfitting procedure, this method converges on a solution balancing data fit with smoothness.

To identify the most relevant explanatory time-series, for each centre we started with a multivariable GAM model including all theoretically relevant variables at lags of 1 to 6 months and autoregressive terms at lags 1 and 2. We limited lags to 6-months based on widespread evidence of declining relevance of antibiotic exposures by time-since exposure, and prior experience that considerations of longer lags lead to problems of concurvity. On the basis of the GAM outputs, an explanatory variable with a specific lag was retained in the model only if its contribution was significant at a $5 \%$ level of probability (identified on contribution charts by the zero line of non-association falling outside of $95 \%$ confidence intervals around the estimate). The process was run iteratively by removing first those variables and lag combinations whose contributions were non-significant before re-running the GAM model on a reduced subset of variables and lags. The process stopped when the model contained only significant contributions of variables and lags. These constituted the restricted set of explanatory variables for entry into MARS analysis.

A further objective of applying the GAM procedure was to determine whether consideration of non-linear associations is justified in terms of improvement in predictive performance. For each explanatory variable (and lag), GAM provides a comparison of the data fit of a nonlinear spline function $(n l)$ with an analysis in which this relationship is restricted to a linear function $(l)$. Significant improvement in goodness of fit over a linear fit is defined by an Ftest, as follows:

$$
F_{0}=\frac{\left(S S R_{l}-S S R_{n l}\right) /\left(p_{n l}-p_{l}\right)}{S S R_{n l} /\left(n-p_{n l}\right)} \sim F_{\left(d f_{n l}-d f_{l}\right),\left(n-p_{n l}\right)}
$$

where; SSR $=$ Sum of squares of residuals, $n=$ number of observations, $p=$ number of parameters, $l=$ linear function, and $n l=$ non-linear spline function. 
704 The null hypothesis that estimates from an enhanced (non-linear) model do not provide a 705 significantly better fit than those from a linear model can be rejected where $F$ exceeds a critical value $(a=0.05)$ from an F-distribution with $\left(p_{n l}-p_{l}, n-p_{n l}\right)$ degrees of freedom.

\section{Step 4. Multivariable model estimation}

After identifying the most likely explanatory variables (and lags), and whether associations with the outcome series were linear or non-linear, we used the MARS procedure (in the SCAB34S Splines module) to obtain an easily interpretable characterization of these associations. ${ }^{47}$ MARS is a non-parametric regression approach suitable for situations of nonlinear associations that can provide more interpretable and interesting empirical results than GAM. Given our research aims, its particular advantages were: (i) the ability to identify distinct threshold values ('knots') of the explanatory variables delimiting regions (ranges of values) of individual or interacting explanatory variables within which associations with the outcome differs substantially from those in other regions; and (ii) a systematic approach for model identification and estimation, which automatically selects the combination of explanatory variables and threshold values which most efficiently explain variation in outcomes. MARS is related to, but more general than, regression tree algorithms, and has several important, interrelated advantages: (a) when thresholds may affect several explanatory variables, the final MARS model is far more synthetic, and therefore more interpretable, in terms of quantifying how each explanatory variable (e.g. antibiotic use) affects the response variable (e.g. resistance) and interacts with other explanatory variables; (b) the MARS algorithm in general deals much better with numerical data and continuous data; (c) it allows for dynamic relationships and thresholds in the dynamic dependences especially important when analysing effects with time series data; and (d) MARS does better in extrapolating the results outside the sample data ranges of explanatory and outcome variables. These advantages are important when considering policy implications of findings for control of AMR.

MARS approximates the functional relationship between an outcome time-series $\left(y_{t}\right)$ and a vector of $p$ explanatory variables $x_{t}=\left(x_{t}^{1} \ldots . . x_{t}^{p}\right)$ as:

where;

$$
y_{t}=\beta_{0}+\sum_{m=1}^{M} \beta_{m} b_{m}\left(x_{t}\right)+\varepsilon_{t}
$$

$\beta_{0}$ is a constant

$\beta_{\mathrm{m}}$ is the coefficient for the $m^{\text {th }}$ basis function, $\mathrm{m}=1, \ldots, \mathrm{M}$

$b_{m}\left(x_{t}\right)$ is the $m^{\text {th }}$ basis function, $\mathrm{m}=1, \ldots, \mathrm{M}$

$\varepsilon_{t}$ is an independently distributed error term.

The basis functions are products of up to two truncated linear or hinge functions, describing the relationship between one or more explanatory variables and the outcome in terms of segments of stable association separated by knots or thresholds values. These interacting hinge functions allow us to identify possible interactions between variables as in Figure $1 C$ (ii). Namely, the $m^{\text {th }}$ basis function takes one of the following two forms:

No interaction: $\quad b_{m}\left(x_{t}\right)=h\left(x_{t}^{k}, t_{k, m}\right)$ for some $k=1, \ldots, p$

With interaction: $b_{m}\left(x_{t}\right)=h_{m}\left(x_{t}^{k}, t_{k, m}\right) \cdot h_{m}\left(x_{t}^{j}, t_{j, m}\right)$ for some $k, j=1, \ldots, p, k \neq j$

747 where $t_{k, m}$ is the threshold value of $x_{t}^{k}$ in the $m^{\text {th }}$ basis function and where $h\left(x_{t}^{k}, t_{k, m}\right)$ is a 748 hinge function that takes the following form depending on whether the basis function takes 749 effect above or below the threshold $t_{k, m}$ 
a) above the threshold: $\quad h_{m}\left(x_{t}^{k}, t_{k, m}\right)=\max \left(x_{t}^{k}-t_{k, m}, 0\right)$

b) below the threshold:

$h_{m}\left(x_{t}^{k}, t_{k, m}\right)=\max \left(t_{k m}-x_{t}^{k}, 0\right)$

If no knot (threshold) is detected, then a simple linear (and therefore constant) association between explanatory and outcome variable can be specified as a single function applied across the total range of values of the explanatory variable.

756

757

758

759

760

All potentially significant explanatory variables, and associated lags, identified in previous steps, were incorporated into models. Model identification and estimation proceed by an automated, iterative, process:

Forward pass: starting with the simplest model containing only a constant basis function, MARS generates a matrix of basis functions in a forward stepwise manner. Candidate base functions are added in order of ability to improve model fit, until the model reaches a predefined limit of complexity. The candidate basis functions are identified by a nested exhaustive search looping over the existing set of basis functions, and all other possible explanatory variables (or interactions) and knot positions.

Backwards (pruning) pass: During the subsequent pruning pass MARS removes basis functions contributing least to model fit, until no significant improvement is seen in a modified form of the generalized cross validation (MGCV) criterion:

$\operatorname{MGCV}=\frac{\frac{1}{N} \sum_{i=0}^{n}(y-f(x))^{2}}{1-((C(M)+d M) / n)^{2}}$

Where; $N$ is the number of observations, $\sum_{i=0}^{n}(y-f(x))^{2}$ is the sum of square of residuals (observed - estimated $y$ ).

$C(M)$ is the number of parameters being fitted, $M$ the number of non-constant basis functions and $d=3$ (conventional value).

The MGCV incorporates a complexity penalty accounting for the inherent improvement in explained variance associated with increasing numbers of basis-functions, and its calculation allows estimates of the relative importance of each basis function. Model selection therefore converges on a set of basis functions that most efficiently explain variation in antibiotic resistance before a final model fit by OLS estimation.

From the output of each MARS model we generated contribution charts illustrating the change in the outcome time-series across the observed ranges of explanatory variables.

\section{Step 5. Diagnostic checks}

Adequacy of model fit was defined by three criteria: (i) Normally distributed residuals - with homogenous variance and mean equal to zero, as evaluated by a Normality test; (ii) absence of significant residual autoregression - identified in lags 0 to 6 on an autocorrelation function (ACF) plot; and (iii) absence of residual non-linearities - as evaluated by a Hinich test. In addition to the MGCV we reported more familiar measures of model performance such as $\mathrm{R}^{2}$ and the mean absolute percentage error (MAPE).

Step 6: Confidence intervals (CIs) for thresholds values 
In the absence of an existing method for deriving measures of uncertainty around thresholds derived from non-parametric MARS models, we develop a procedure inspired by Hansen (2000) ${ }^{48}$ His procedure considers a simple threshold model with only one variable affected by a threshold effect, and obtains a distribution theory for the threshold parameter $(\tau)$ from which asymptotic confidence intervals can be built. He first derives the limiting distribution of a Likelihood Ratio test (LR) for the null hypothesis that the threshold parameter $\tau=\tau_{0}$. He then builds confidence intervals through the inversion of LR: the (1- $\alpha)$ Inverted Likelihood Ratio (ILR) confidence interval consisting of all the possible values of $\tau$ for which the null hypothesis would not be rejected at the $\alpha$ level. Donayre et al. (2018) examine improvements of Hansen's ILR confidence interval, increasing its quality in finite samples with large threshold effects (i.e. when the change in slope from one side of the threshold to the other is large). ${ }^{49}$ They show that a 'conservative modification' enlarging Hansen's ILR confidence interval is optimal. In this "conservative ILR confidence interval" the lower end of the interval is enlarged from the first value lower than $\tau_{l}$ for which the null hypothesis is rejected, up to $\tau_{l}$; at the upper end, it is enlarged from $\tau_{u}$ up to the first value greater than $\tau_{u}$ for which the null hypothesis is rejected. This modification provides intervals at a confidence level at least as high as the nominal one that are still informative.

We adapted this procedure for MARS estimations with more than one explanatory variable containing thresholds, and one or more thresholds per variable, by using the partial residuals -i.e. the variation in the outcome not explained by other explanatory variables and their thresholds. This allowed us to identify conservative ILR confidence intervals for each explanatory variable, conditional on all the estimated coefficients and thresholds other than the one for which the confidence interval is computed. Since in MARS all thresholds and slope coefficients are anyway selected and estimated to optimise overall model fit using conditional inference, identifying these 'conditional conservative ILR (CCILR) confidence intervals' does not impose costs to reliability. Computing confidence intervals conditional only on other thresholds, but with re-estimation of coefficients describing piece-wise associations (slopes), offers a valid alternative. In Monte Carlo simulations (results available on request) we found both approaches resulted in adequate coverage ( $>95 \%$ of intervals including the actual value of the threshold) but CIs were wider with a procedure with slope re-estimation and less informative for a given coverage rate. Our simulations show that the superiority of the partial residual approach is independent of the degree of correlation between antibiotic segments (values of antibiotic use over which association with the outcome is constant) where correlation is between $0 \%$ and $75 \%$. There is some risk of very slight under coverage with the partial residual approach where correlations are $\geq 85 \%$, but such high levels of correlation are unlikely unless several the functional relationship involves multiple, closely occurring, thresholds. In such situations, re-estimation of slope co-efficients is anyway not feasible. As a result, we recommend the partial residual approach, with a caution that the CI could be somewhat wider in specific and infrequent situations where multiple close thresholds for the same independent variable are detected (e.g. fluoroquinolone association with \%Ec-ESBL in Orihuela hospital population).

Step 7. Interpretation

The minimum thresholds identified for each significantly associated antibiotic group were translated into suggested maximum numbers of patient treatment courses per month not expected to adversely affect resistance at population levels. We multiplied the threshold, expressed in DDDs per 1000 OBDs (or IDs), by the size of the monthly patient population (in thousands of OBDs or IDs), and then divided by an average patient treatment course of 7 DDDs (except for aminoglycosides which were considered as 3 DDDs). These maximums were further compared to contemporary levels of antibiotic use in the last year of study, to 
provide indications of how current use of antibiotics should be modified to avoid resistance spread.

\section{Projections for alternative antibiotic stewardship policy options}

851 To illustrate the potential effects of restricting antibiotics associated with \%Ec-ESBL+ to 852 threshold levels in populations of Orihuela, we compared the expected evolution of \%EcESBL+ under a 'business as usual' scenario in which antibiotic use continued as in last year of study, to projected time-series with antibiotics restricted to threshold levels. We used a breakpoint analysis to identify the last stationary segment in \%Ec-ESBL+ time-series from the study period. We recursively estimated MARS models using means from these stationary segments as starting points to derive steady states for \%Ec-ESBL+ in community and hospital populations. Based on steady state values and MARS models for the study period 859 (baseline) we simulated 1000 samples of 24-month projections, incorporating random error 860 term with variance as derived in the baseline MARS model. For each sample projection we 861 entered mean antibiotic levels in the last year of the study period ('business as usual') and alternative levels set at identified thresholds. We calculated the mean difference between business as usual and each policy option for every month along with $95 \%$ confidence intervals. Finally, we illustrated alternative projections and differences using medians of distributions from the 1,000 sample projections.

\section{Data availability}

869 The data that support the findings of this study are available from the corresponding author upon reasonable request. 


\section{References}

1. Laxminarayan, R. Antibiotic effectiveness: Balancing conservation against innovation. Science 345, 1299-301 (2014)

2. Laxminarayan, R., et al. Antibiotic resistance - the need for global solutions. Lancet Infect Dis. 13, 1057-98 (2013)

3. World Bank. Drug-Resistant Infections: A Threat to Our Economic Future (discussion draft). World Bank, Washington DC. Available from: http://pubdocs.worldbank.org/en/689381474641399486/1701381-AMR-Lab-Report-Web.pdf (2016).

4. O’Neill, J. (chair). Review on Antimicrobial Resistance. Antimicrobial Resistance: Tackling a Crisis for the Health and Wealth of Nations. Available from: https://amrreview.org/ (2014)

5. Millar, M. Constraining the use of antibiotics: applying Scanlon's contractualism. $J$ Med Ethics. 38, 465-9 (2012)

6. WHO. Global action plan on antimicrobial resistance. World Health Organization, Geneva, Switzerland. Available from: http://www.who.int/antimicrobialresistance/publications/global-action-plan/en/ (2015).

7. Davey, P., et al. Interventions to improve antibiotic prescribing practices for hospital inpatients. Cochrane Database of Systematic Reviews 2, CD003543. (2017)

8. Peterson, L.R., Squeezing the antibiotic balloon: the impact of antimicrobial classes on emerging resistance. Clin Microbiol Infect. 2005 Oct;11 Suppl 5:4-16.

9. Levy, S.B. Balancing the drug-resistance equation. Trends Microbiol. 2, 341-2 (1994)

10. Levin, B.R., et al. The Population Genetics of Antibiotic Resistance. Clin Infect Dis. 24(Supplement 1), S9-16 (1997)

11. Austin, D.J., Kristinsson, K.G. \& Anderson, R.M. The relationship between the volume of antimicrobial consumption in human communities and the frequency of resistance. Proc Natl Acad Sci. 96, 1152-6 (1999). 
944 12. López-Lozano, J.M., et al. Modelling and forecasting antimicrobial resistance and its 945 dynamic relationship to antimicrobial use: a time series analysis. Int J Antimicrob Agents. 14, 946 21-31 (2000).

947

13. Vernaz, N., et al. Modelling the impact of antibiotic use on antibiotic-resistant Escherichia coli using population-based data from a large hospital and its surrounding community. J Antimicrob Chemother. 66(4), 928-35 (2011).

14. Aldeyab, M.A., et al. Modelling the impact of antibiotic use and infection control practices on the incidence of hospital-acquired methicillin-resistant Staphylococcus aureus: a time-series analysis. J Antimicrob Chemother. 62, 593-600 (2008).

15. Monnet, D.L., et al. Antimicrobial drug use and methicillin-resistant Staphylococcus aureus, Aberdeen, 1996-2000. Emerg Infect Dis. 10, 1432-41 (2004).

16. Aldeyab, M.A., et al. The impact of antibiotic use on the incidence and resistance pattern of extended-spectrum beta-lactamase-producing bacteria in primary and secondary healthcare settings. Br J Clin Pharmacol. 74,171-9 (2012).

17. Haber, M., Levin, B.R. \& Kramarz, P. Antibiotic control of antibiotic resistance in hospitals: a simulation study. BMC Infect Dis. 10, 254 (2010)

18. Oz T, Guvenek A, Yildiz S, et al. Strength of Selection Pressure Is an Important Parameter Contributing to the Complexity of Antibiotic Resistance Evolution. Molecular Biology and Evolution. 31(9), 2387-2401 (2014).

19. Arepyeva, M.A., et al. A mathematical model for predicting the development of bacterial resistance based on the relationship between the level of antimicrobial resistance and the volume of antibiotic consumption. J Glob Antimicrob Resist. 8,148-156 (2017).

20. Kiffer, C.R., et al. A spatial approach for the epidemiology of antibiotic use and resistance in community-based studies: the emergence of urban clusters of Escherichia coli quinolone resistance in Sao Paulo, Brasil. Int J Health Geogr. 10, 17 (2011).

21. Vogwill, T. \& MacLean, R.C. The genetic basis of the fitness costs of antimicrobial resistance: a meta-analysis approach. Evol Appl. 8, 284-95 (2015).

22. Berger, P., et al. Generalized Additive Model Demonstrates Fluoroquinolone Use/Resistance Relationships for Staphylococcus aureus. Eur J Epidemiol. 19, 453-60 (2004).

23. Lawes, T., et al. Effects of national antibiotic stewardship and infection control strategies on hospital-associated and community-associated meticillin-resistant Staphylococcus aureus infections across a region of Scotland: a non-linear time-series study. Lancet Infect Dis. 15,1438-49 (2015).

24. Lawes, T., et al. Turning the tide or riding the waves? Impacts of antibiotic stewardship and infection control on MRSA strain dynamics in a Scottish region over 16 years: non-linear time series analysis. BMJ Open, 5, e006596 (2015). 
994

995

996

997

998

999

1000

1001

1002

1003

1004

1005

1006

1007

1008

1009

1010

1011

1012

1013

1014

1015

1016

1017

1018

1019

1020

1021

1022

1023

1024

1025

1026

1027

1028

1029

1030

1031

1032

1033

1034

1035

1036

1037

1038

1039

1040

1041

1042

1043

25. Lawes, T., et al. Effect of a national 4C antibiotic stewardship intervention on the clinical and molecular epidemiology of Clostridium difficile infections in a region of Scotland: a nonlinear time-series analysis. Lancet Infect Dis. 17, 194-206 (2017).

26. Wong A. Epistasis and the Evolution of Antimicrobial Resistance. Front Microbiol. 8, 246 (2017).

27. Levin BR, Perrot V, Walker N. Compensatory mutations, antibiotic resistance and the population genetics of adaptive evolution in bacteria. Genetics 154, 985-97 (2000).

28. Andersson DI, Hughes D. Antibiotic resistance and its cost: is it possible to reverse resistance? Nat Rev Microbiol. 8(4), 260-71 (2010).

29. Mózes J, Ebrahimi F, Gorácz O, Miszti C, Kardos G. Effect of carbapenem consumption patterns on the molecular epidemiology and carbapenem resistance of Acinetobacter baumannii. J Med Microbiol. 63, 1654-62 (2014).

30. Díaz, M.A., et al. Diversity of Escherichia coli Strains Producing Extended-Spectrum $\beta$ Lactamases in Spain: Second Nationwide Study. J Clin Microbiol. 48(8), 2840-5 (2010).

31. Rodríguez-Baño J, Alcalá JC, Cisneros JM, Grill F, Oliver A, Horcajada JP, Tórtola T, Mirelis B, Navarro G, Cuenca M, Esteve M, Peña C, Llanos AC, Cantón R, Pascual A. Community infections caused by extended-spectrum beta-lactamase-producing Escherichia coli. Arch Intern Med. 168(17), 1897-902 (2008).

32. Branger, C., et al. Genetic Background of Escherichia coli and Extended-spectrum $\beta$ Lactamase Type. Emerg Infect Dis. 11(1), 54-61 (2005).

33. Rodríguez-Baño, J., et al. Impact of changes in CLSI and EUCAST breakpoints for susceptibility in bloodstream infections due to extended-spectrum $\beta$-lactamase-producing Escherichia coli. Clin Microbiol Infect Dis.18(9), 894-900 (2012).

34. Poole, K. Pseudomonas aeruginosa: Resistance to the Max. Frontiers in Microbiology 2, 65. doi:10.3389/fmicb.2011.00065 (2011).

35. Dubois, V., et al. Beta-lactam and aminoglycoside resistance rates and mechanisms among Pseudomonas aeruginosa in French general practice (community and private healthcare centres). J Antimicrob Chemother. 62:316-23 (2008).

36. Hocquet, D., et al. Relationship between antibiotic use and incidence of MexXY-OprM overproducers among clinical isolates of Pseudomonas aeruginosa. Antimicrob Agents Chemother. 52, 1173-5 (2008).

37. Horváth, A., et al. Varying fitness cost associated with resistance to fluoroquinolones governs clonal dynamic of methicillin-resistant Staphylococcus aureus. Eur J Clin Microbiol Infect Dis 31:2029-3 (2012)

38. Laxminarayan, R., \& Brown, G.M. Economics of Antibiotic Resistance: A Theory of Optimal Use. Journal of Environmental Economics and Management 42, 183-206 (2001). 
1044

1045

1046

1047

1048

1049

1050

1051

1052

1053

1054

1055

1056

1057

1058

1059

1060

1061

1062

1063

1064

1065

1066

1067

1068

1069

1070

1071

1072

1073

1074

1075

1076

1077

1078

1079

1080

1081

1082

1083

1084

1085

1086

1087

1088

1089

1090

1091
39. The Health Foundation. On targets: How targets can be most effective in the English NHS. the Health Foundation, London (2015).

40. Hulscher, M.E., Grol, R.P., van der Meer J.W. Antibiotic prescribing in hospitals: a social and behavioural scientific approach. Lancet Infect Dis., 10(3)167-75. (2010)

41. Laxminarayan, R., \& Klugman, K.P. Communicating trends in resistance using a drug resistance index. BMJ Open 1, e000135 (2011).

42. Hughes, J.S., Hurford, A., Finley, R.L., et al. How to measure the impacts of antibiotic resistance and antibiotic development on empiric therapy: new composite indices. BMJ Open 6, e012040. (2016)

43. Murphy, T.E., Van Ness, P.H., Araujo, K.L.B., Pisani, M.A. Bayesian Time-Series Analysis of a Repeated-Measures Poisson Outcome With Excess Zeroes. American Journal of Epidemiology 174(11), 1230-1237 (2011)

44. Søgaard P. The epidemiology of antibiotic resistance in three species of the Enterobacteriaceae and the relation to consumption of antimicrobial agents in Odense University Hospital. Dan Med Bull.36(1):65-84 (1989).

45. Møller JK. Antimicrobial usage and microbial resistance in a university hospital during a seven-year period. J Antimicrob Chemother.24(6):983-92 (1989).

46. Hastie, T. \& Tibshirani, R. Generalized Additive Models. Statist. Sci. 1, 297-310 (1986)

47. Friedman, J.H. Multivariate Adaptive Regression Splines. Ann. Statist 19, 1-67 (1991)

48. Hansen, B.E. Sample splitting and threshold estimation. Econometrica 68(3), 575-603 (2000).

49. Donayre, L., Eo, Y., Morley, J. Improving likelihood-ratio-based confidence intervals for threshold parameters in finite samples. Studies in Nonlinear Dynamics and Econometrics, 22(1), Article number 20160084 (2018). 
1092

1093

1094

1095

1096

1097

1098

1099

1100

1101

1102

1103

1104

1105

1106

1107

1108

1109

1110

1111

1112

1113

1114

1115

1116

1117

1118

1119

1120

1121

1122

1123

1124

1125

1126

1127

1128

1129

1130

1131

1132

1133

1134

1135

1136

1137

1138

1139

\section{Additional authors in THRESHOLDS study group}

María Núñez-Núñez ${ }^{14}$, Ana I. Suárez ${ }^{14}$, Michelle Thouverez ${ }^{5}$, Navarro Cots ${ }^{15}$, Emilio Borrajo $^{15}$, Carlos Devesa ${ }^{15}$, Joan Gregori ${ }^{15}$, Inmaculada García Cuello ${ }^{15}$, Isabel Pacheco ${ }^{15}$, María Cerón ${ }^{15}$, Hajnalka Tóth ${ }^{16}$.

14. Hospital Virgen de la Macarena, Sevilla, Spain

15. Hospital Vega Baja, Orihuela, Spain

16. Department of Medical Microbiology, Faculty of Medicine, University of Debrecen, Debrecen, Hungary

\section{Supplementary information}

Is available in the online version of the article

Correspondence and requests for data, R-scripts or other study materials can be addressed to

Dr Timothy Lawes, t.lawes@nhs.net

\section{Acknowledgements}

All authors acknowledge Dominique L. Monnet, from the European Centre for Disease Prevention and Control (ECDC) for his continuous support, guidance and intellectual contributions to the THRESHOLDS project. J-M.L-L., acknowledges the continuous support of the Management Team of the Hospital Vega Baja, and the technical support of Carlos Quiles (Cabosoft SL), in the development of time-series analysis techniques in the field of antimicrobial resistance, and in particular the WebResist project (www.webresist.org) which provided a framework for the development of this study. G.K., was supported by a Bolyai Research Scholarship of the Hungarian Academy of Sciences. T.L., was supported by the Wellcome Trust. J.R-B., and P.R., received funding for research from Plan Nacional de I+D+i 2013-2016 and Instituto de Salud Carlos III, Subdirección General de Redes y Centros de Investigación Cooperativa, Ministerio de Economía, Industria y Competitividad, Spanish Network for Research in Infectious Diseases (REIPI RD16/0016/0001) - co-financed by European Development Regional Fund "A way to achieve Europe", Operative Programme Intelligent Growth 2014-2020.

\section{Author contributions}

J-M.L-L., T.L., C.N., A.B., and I.M.G., proposed the original idea and designed the study. X.B., D.H., M.A., G.C-B., M.S., D.F., G.K., J.R-B., P.R., and N.G., collated centre-specific data, situational analysis, and hypotheses. J-M.L-L., T.L., C.N., A.B., contributed to statistical analysis, with C.N. and A.B, the principal analysts. All authors discussed the results and commented on the manuscript.

\section{Competing interests}

IMG is in receipt of payments for consultancies and lectures from numerous Pharmaceutical firms developing new antimicrobials. Otherwise, the authors declare no competing interests. 


\section{Figure legends}

Figure 1 | Carbapenem-resistant $A$. baumannii and antibiotic use

(a) Time series for observed and predicted incidence density of CRAb, with observed incidence density of CSAb. (b) Time series for use of potential explanatory antibiotic groups (5-month moving averages). (c) Contribution charts illustrating the relationship between explanatory variables and CRAb incidence density (sample size, $\mathrm{n}=143$ months of observation).

${ }^{a}$ Change relative to median monthly CRAb incidence density for study period. CRAb, carbapenem-resistant A. baumannii. CSAb, carbapenem-susceptible A. baumannii. DDDs, defined daily doses. IQR, Interquartile range. MARS, Multivariate Adaptive Regression Splines. NL-TSA, non-linear time series analysis. OBDs, occupied bed days. antibiotic use in hospital and community.
(a) Time series for observed and predicted \%Ec-ESBL+ in hospital population. (b) Time series of potential explanatory antibiotic groups in hospital population (5-month moving averages). (c) Time series for observed and predicted \%Ec-ESBL+ in community population. (d) Time series of potential explanatory antibiotic groups in community population (5-month moving averages). (e) Contribution charts illustrating the relationship between explanatory variables and hospital or community $\%$ Ec-ESBL+. (sample size, $n=304$ months of observation).

${ }^{a}$ compared to median monthly \%Ec-ESBL+ for study period.

* confidence interval around lower threshold in association between fluoroquinolones and $\%$ Ec-ESBL + in hospital may be wider due to risk of slight under-coverage of confidence interval estimation arising from a problem of multiple closely occurring thresholds.

$\%$ Ec-ESBL+, percentage of $E$. coli isolates producing extended-spectrum beta-lactamases. DDDs, defined daily doses. IQR, Interquartile range. MARS, Multivariate Adaptive Regression Splines. NL-TSA, non-linear time series analysis. OBDs, occupied bed days.

\section{Figure 3 | Cefepime-resistant Escherichia coli and antibiotic use}

(a) Time series for observed and predicted incidence density of \%Ec-FepR. (b) Time series of potential explanatory antibiotic groups (5-month moving averages). (c) Contribution charts illustrating the relationship between explanatory variables and \%Ec-FepR (sample size, $\mathrm{n}=105$ months of observation).

${ }^{a}$ compared to median monthly $\%$ Ec-FepR for study period.

DDDs, Defined Daily Doses. IQR, interquartile range. \%Ec-FepR, percentage of E. coli isolates resistant to cefepime. OBDs, Occupied Bed Days.

\section{Figure 4: Gentamicin-resistant Pseudomonas aeruginosa and antibiotic use}

(a) Time series for observed and model predicted incidence density of GRPa. (b) Time series of potential explanatory antibiotic groups (5-month moving averages) (c) Contribution charts illustrating the relationship between explanatory variables and GRPa incidence density

a compared to median monthly GRPa incidence density for study period (sample size, $\mathrm{n}=192$ months of observation).

DDDs, Defined Daily Doses. GRPa, gentamicin-resistant P. aeruginosa; GSPa, gentamicinsusceptible $P$. aeruginosa isolates. IQR, Interquartile range. MARS, multivariate adaptive regression splines OBDs, Occupied Bed Days. 
Figure 5: Methicillin-resistant Staphylococcus aureus, hand hygiene, and antibiotic use

(a) Time series for observed and predicted incidence density of MRSA. (b) Time series of potential explanatory antibiotic groups (5-month moving averages). (c) Time series for alcohol-based hand rub (ABHR) use. (d) Contribution charts illustrating the relationship between explanatory variables and MRSA incidence density (sample size, $n=105$ months of 1195 observation).

$1196{ }^{a}$ compared to median monthly MRSA incidence density for study period.

1197 ABHR, alcohol-based hand rub. DDDs, Defined Daily Doses. ECDC, European Centre for 1198 Disease Prevention and Control. MRSA, Methicillin-resistant Staphylococcus aureus. OBDs, 1199 Occupied Bed Days. 
Table 1: Translation of thresholds identified in non-linear models into populationspecific antibiotic stewardship policy recommendations

Patient treatments per month

\begin{tabular}{|c|c|c|c|c|}
\hline \multirow[b]{3}{*}{ Antibiotic } & \multirow[b]{3}{*}{$\begin{array}{c}\text { Maximum suggested } \\
\text { by threshold } \\
(95 \% \mathrm{CI})\end{array}$} & \multirow{3}{*}{$\begin{array}{l}\text { Average use } \\
\text { in last } 12 \\
\text { months of } \\
\text { study }\end{array}$} & \multirow{2}{*}{\multicolumn{2}{|c|}{ Suggested reduction in use $(\%)$}} \\
\hline & & & & \\
\hline & & & $\begin{array}{c}\text { Standard } \\
\text { (using point estimate for } \\
\text { threshold) }\end{array}$ & $\begin{array}{c}\text { Conservative } \\
\text { (using lower limit of } 95 \% \\
\text { CI for threshold) }\end{array}$ \\
\hline \multicolumn{5}{|c|}{ 1. Carbapenem-resistant Acinetobacter baumannii (CRAb)\$ Debrecen, Hungary } \\
\hline Carbapenems & $86(45$ to 134$)$ & 226 & $140(62 \%)$ & $181(80 \%)$ \\
\hline $3^{\text {rd }}$ generation cephalosporins & $203(169-229)$ & 299 & $96(32 \%)$ & $130(57 \%)$ \\
\hline Fluoroquinolones & $478(367-576)$ & 375 & Maintain below threshold & $9(2 \%)$ \\
\hline Piperacillin-tazobactam & $39(28-78)$ & 41 & $2(5 \%)$ & $13(32 \%)$ \\
\hline \multicolumn{5}{|c|}{ 2. Extended-spectrum $\beta$-lactamase producing Escherichia coli (\%Ec-ESBL+) Orihuela, Spain } \\
\hline \multicolumn{5}{|c|}{ a) Hospital population } \\
\hline Fluoroquinolones & $78(67 \text { to } 96)^{\mathrm{b}}$ & 165 & $68(41 \%)$ & $87(53 \%)^{\mathrm{b}}$ \\
\hline Third-generation cephalosporins & $62(35$ to 91$)$ & 78 & $16(21 \%)$ & $43(55 \%)$ \\
\hline \multicolumn{5}{|l|}{ b) Community population } \\
\hline Fluoroquinolones & 80 (40 to 148$)$ & 66 & Maintain below threshold & $26(39 \%)$ \\
\hline Co-amoxiclav & 191 (142 to 284$)$ & 277 & $86(31 \%)$ & $135(49 \%)$ \\
\hline \multicolumn{5}{|c|}{ 3. Cefepime-resistant Escherichia coli (\%EcFepR) Seville, Spain } \\
\hline Fluoroquinolones & $392(225$ to 426$)$ & 351 & Maintain below threshold & $126(36 \%)$ \\
\hline $3^{\text {rd }} / 4^{\text {th }}$ generation cephalosporins & $130(83$ to 158$)$ & 211 & $87(41 \%)$ & $128(61 \%)$ \\
\hline \multicolumn{5}{|c|}{ 4. Gentamicin-resistant Pseudomonas aeruginosa (GRPa), France } \\
\hline Gentamicin and tobramycin & $75(36-99)^{\mathrm{b}}$ & $68^{\mathrm{c}}$ & Maintain below threshold & $32(47 \%)$ \\
\hline Fluoroquinolones & $324(316$ to 330$)$ & 223 & Maintain below threshold & Maintain below threshold \\
\hline \multicolumn{5}{|c|}{ 5. Methicillin-resistant Staphylococcus aureus (MRSA) Antrim, Northern Ireland } \\
\hline Fluoroquinolones & $24(20$ to 29$)$ & 39 & $15(39 \%)$ & $19(49 \%)$ \\
\hline $3^{\text {rd }}$ generation cephalosporins & $7(6$ to 8$)$ & 6 & Maintain below threshold & Maintain below threshold \\
\hline Co-amoxiclav & 320 (189 to 422$)$ & 320 & Maintain below threshold & $131(41 \%)$ \\
\hline \multicolumn{5}{|c|}{$\begin{array}{l}\text { a Derived by multiplying the threshold in table 1, expressed in DDDs per } 1000 \text { OBDs, by the size of the population (in } 1000 \text { OBDs or IDs), } \\
\text { and then dividing by an average patient treatment (considered as } 7 \text { DDDs unless otherwise specified) } \\
\text { b Confidence intervals may be wider, and the recommended restriction greater in the conservative approach, due to risk of slight } \\
\text { undercoverage of estimated confidence intervals due to multiple closely occurring thresholds in association between fluoroquinolone and } \\
\text { \%Ec-ESBL+ in hospital. } \\
\text { c Average treatment course considered as } 3 \text { DDDs. }\end{array}$} \\
\hline
\end{tabular}




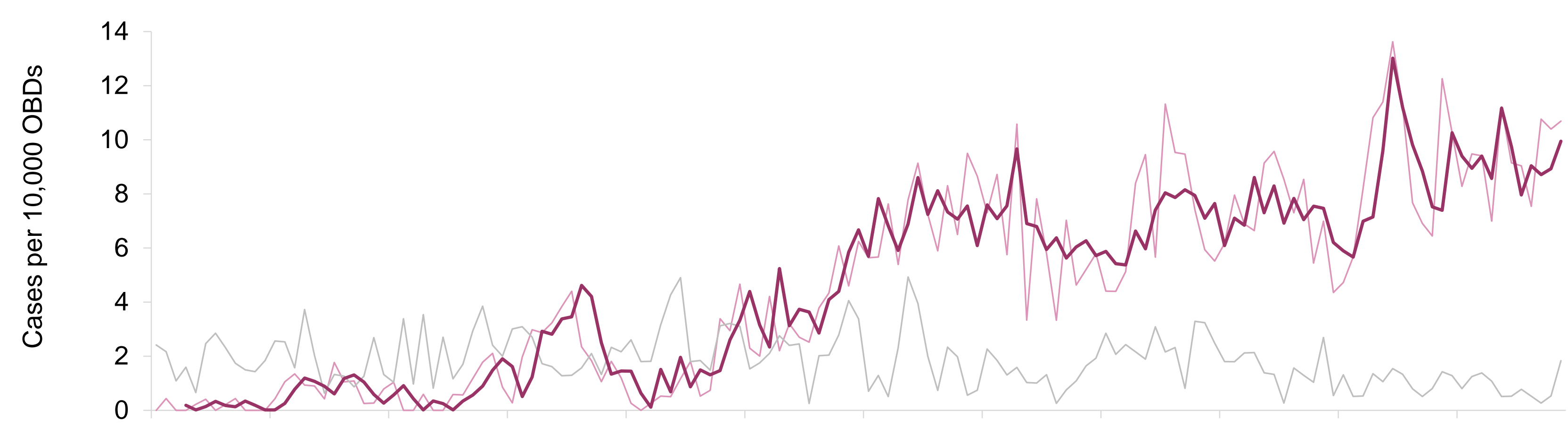

- Observed CRAb

- Observed CSAb

-NL-TSA model (MARS)

b

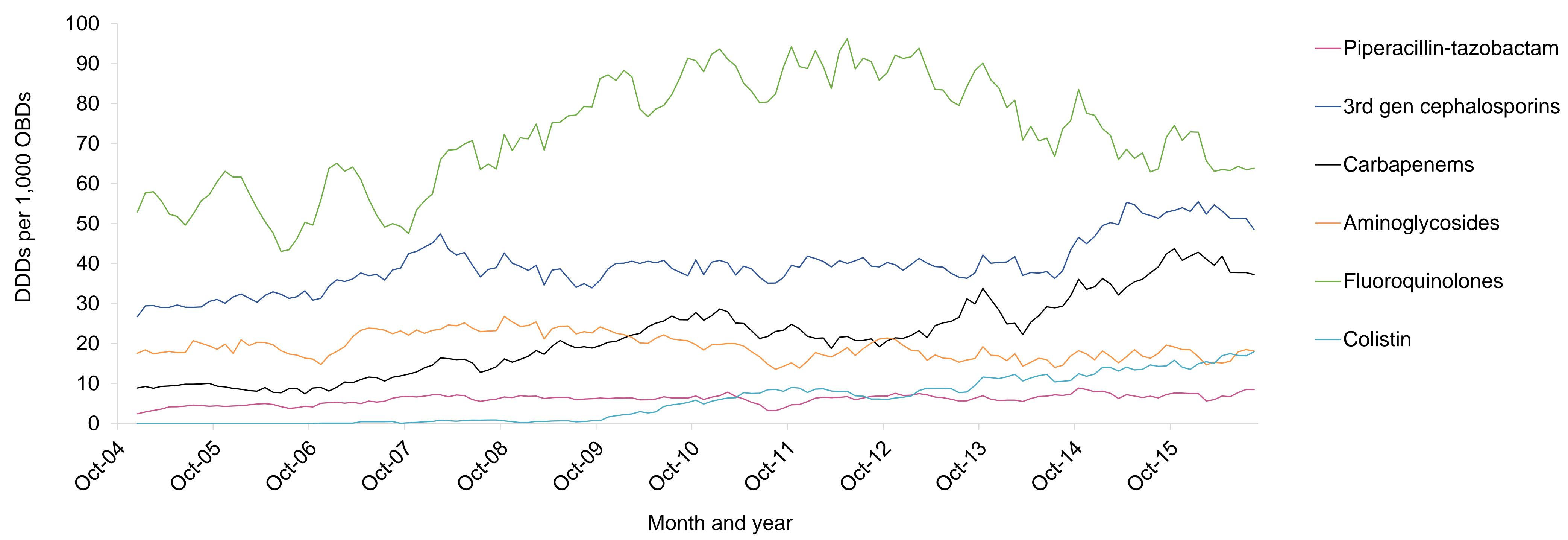

C

Median (IQR) and range of monthly antibiotic use

Estimated change in outcome by level of explanatory variable

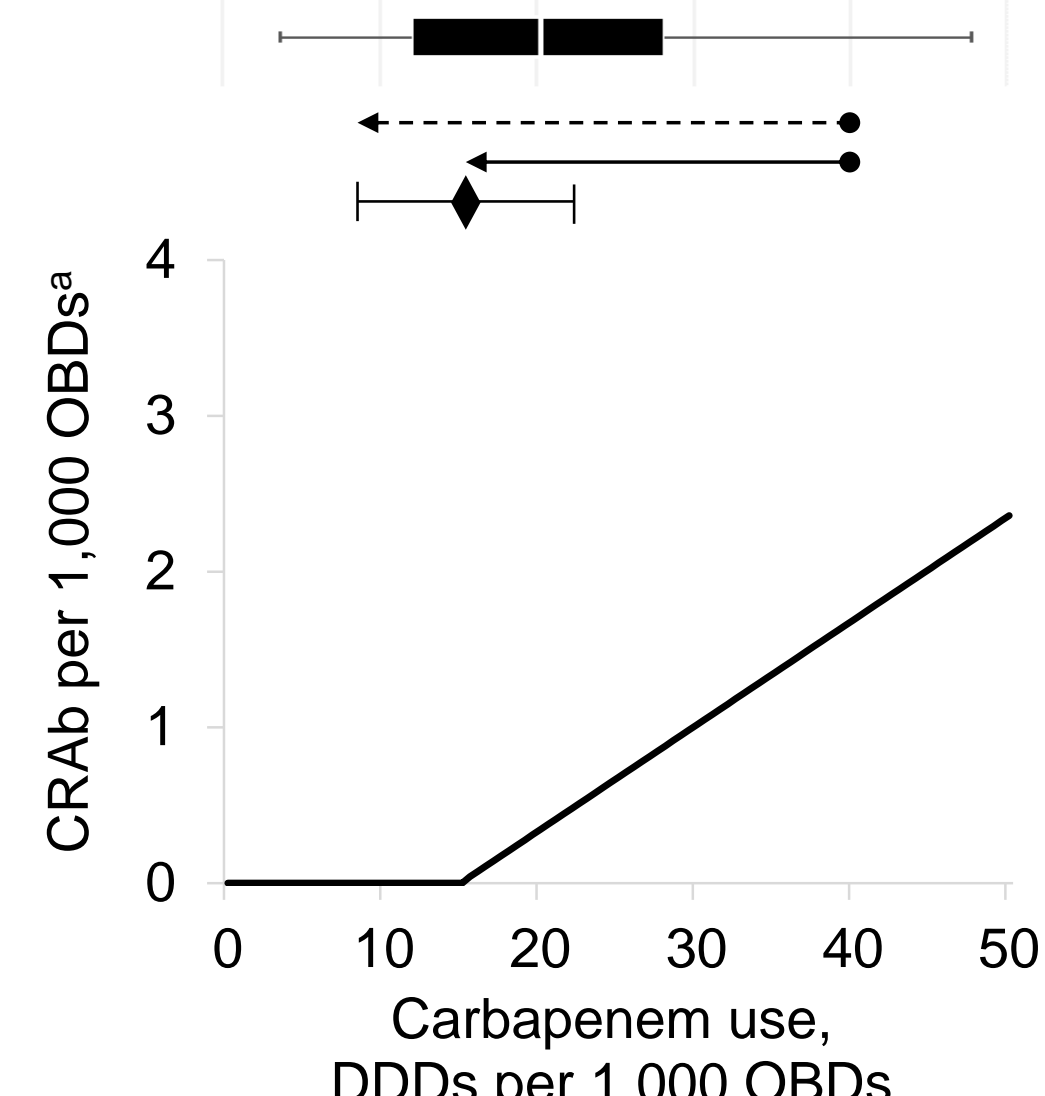

DDDs per 1,000 OBDs
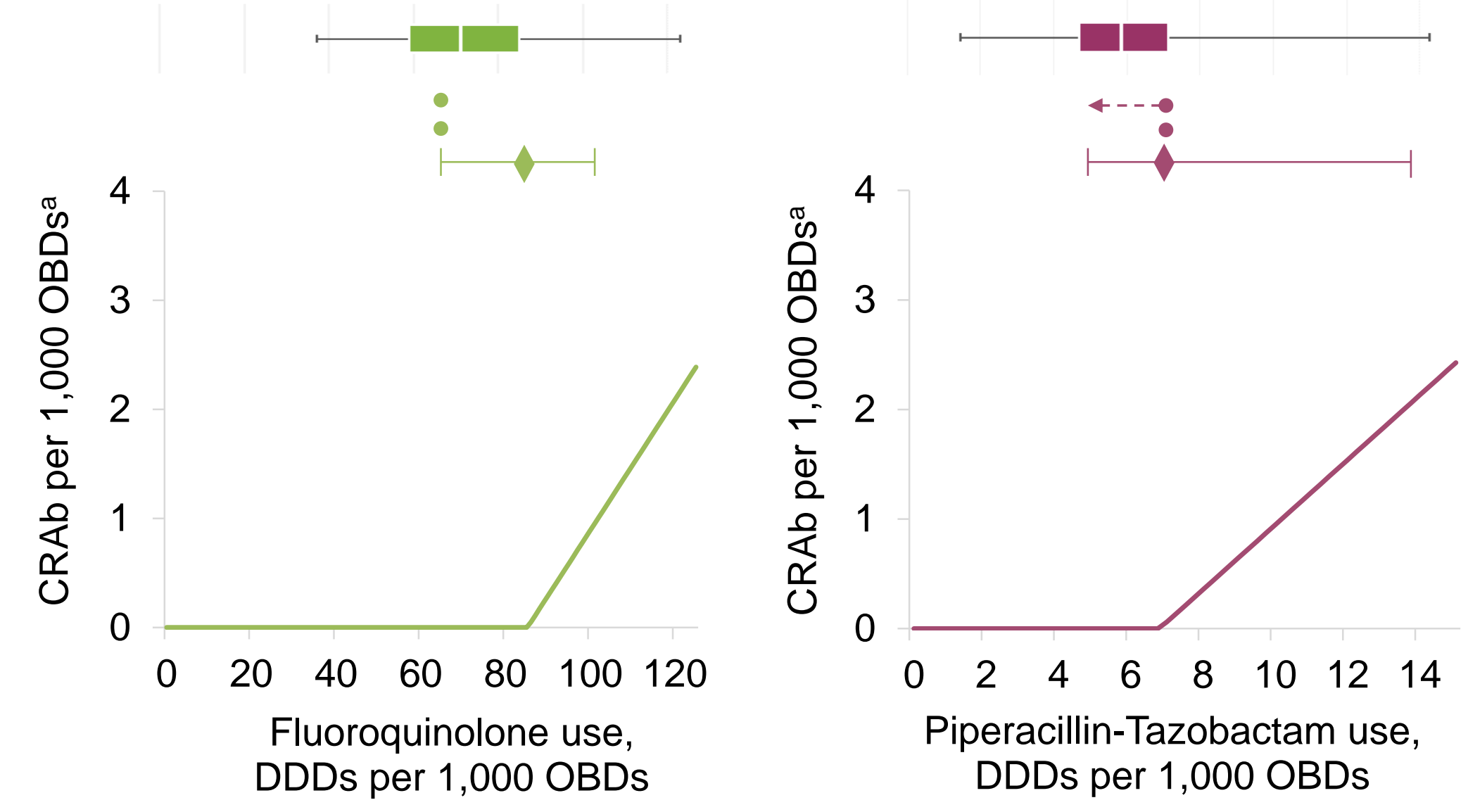


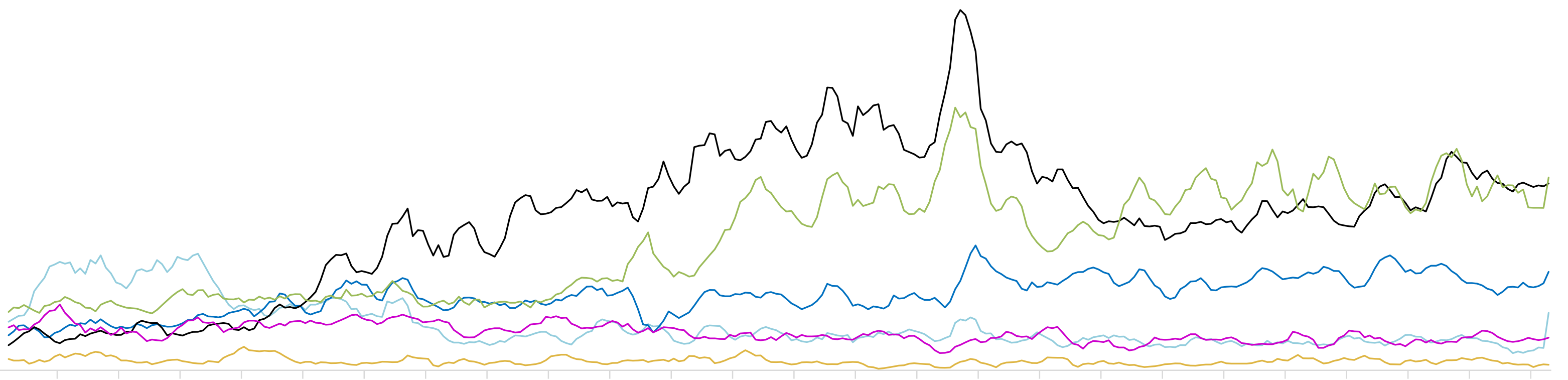

—2nd gen cephalosporins

— 3rd gen cephalosporins

- Coamoxiclav

—Cotrimoxazole

-Aminoglycosides

-Fluoroquinolones

c

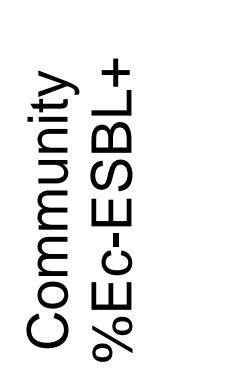

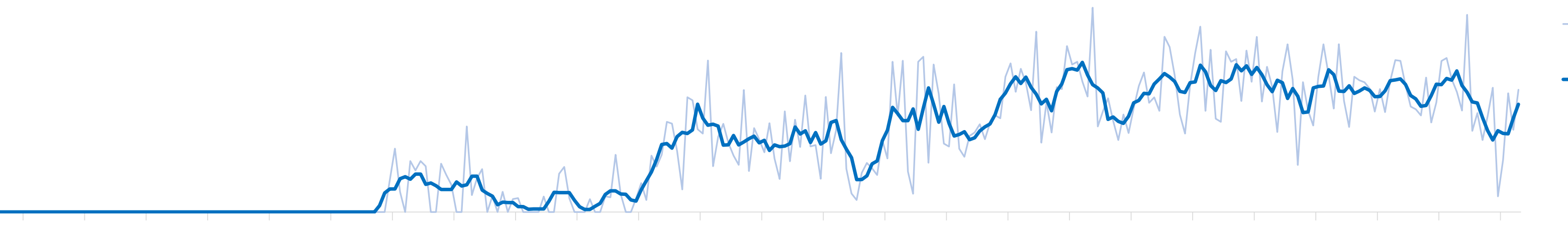

Observed \%ESBL

- Non-linear TSA model

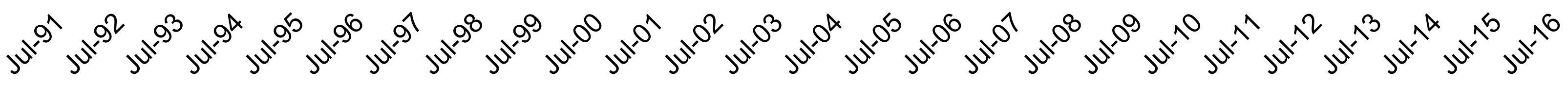

\section{-Fluoroquinolones \\ 2nd gen cephalosporins \\ —3rd gen cephalosporins \\ - Cotrimoxazole \\ -Co-amoxiclav}

Year and month

Median (IQR) and range of monthly antibiotic use

Estimated change in outcome by level of explanatory variable

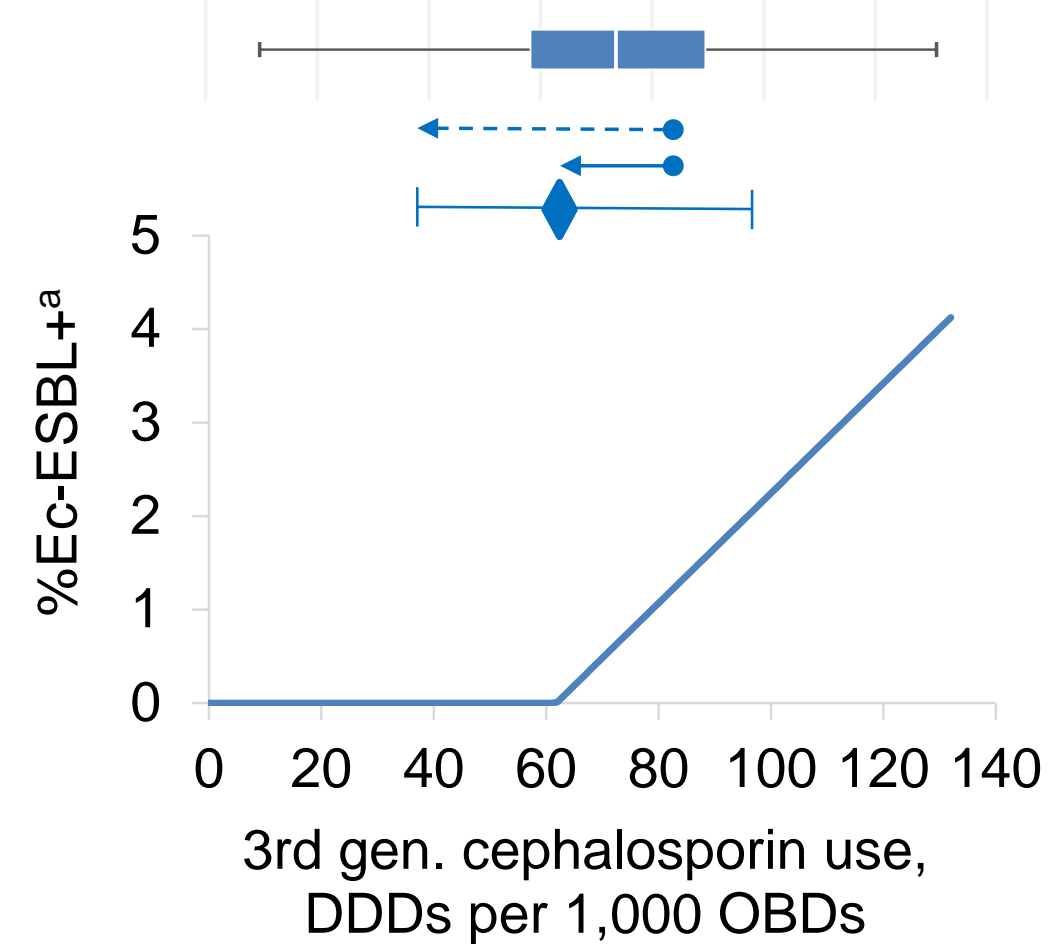

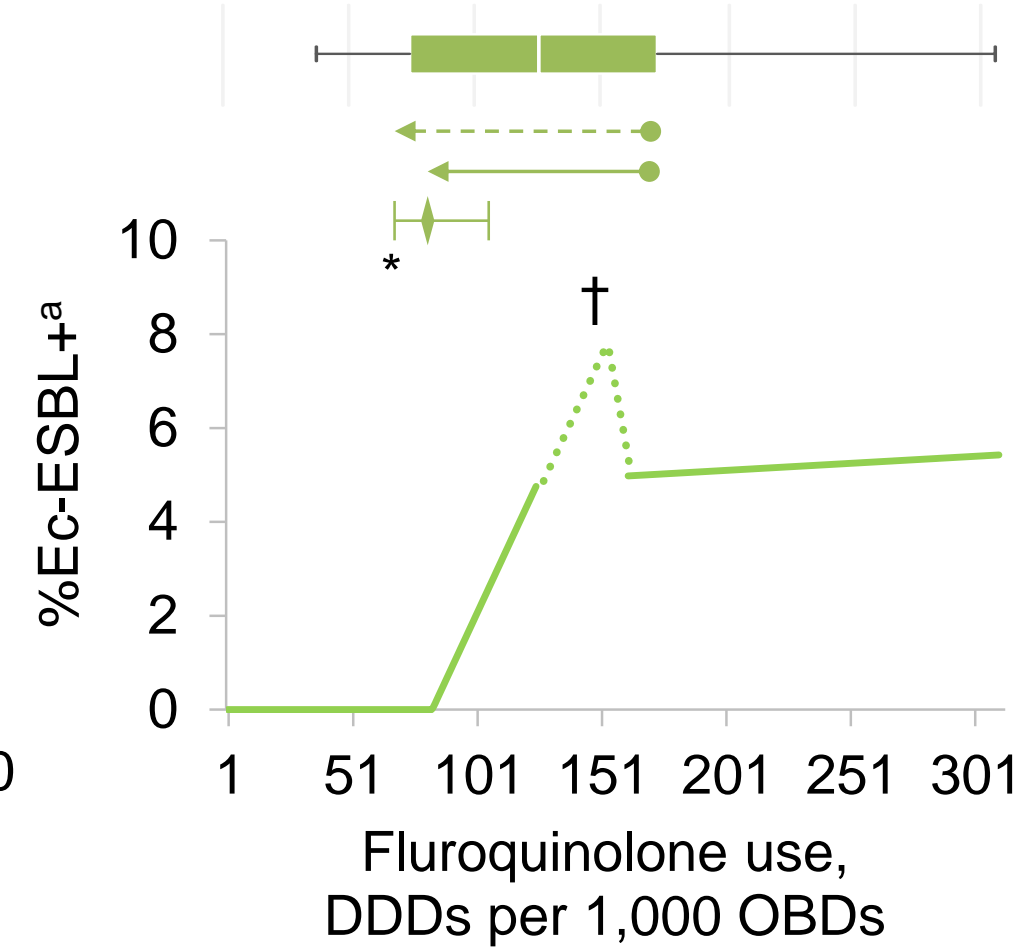

$\vdash \quad$ Threshold and 95\% confidence interval

- Current use level (circle) and suggested change in use for standard (solid arrow) or conservative (dashed arrow) policy options

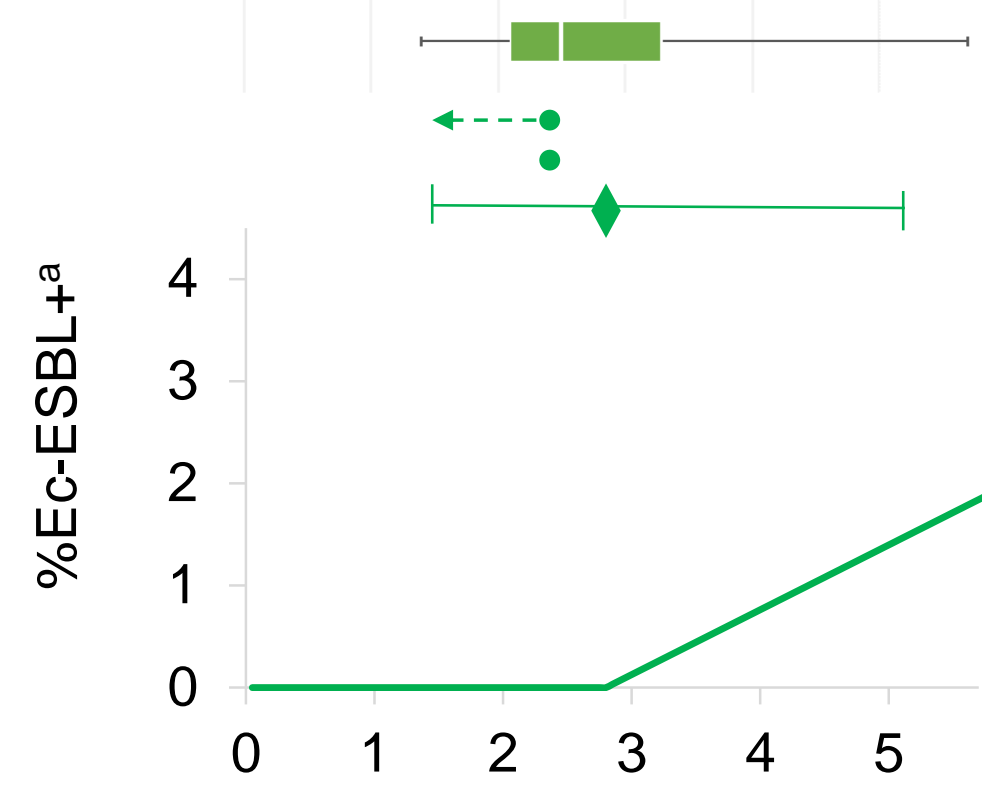

Fluroquinolone use (community), DDDs per 1,000 IDs

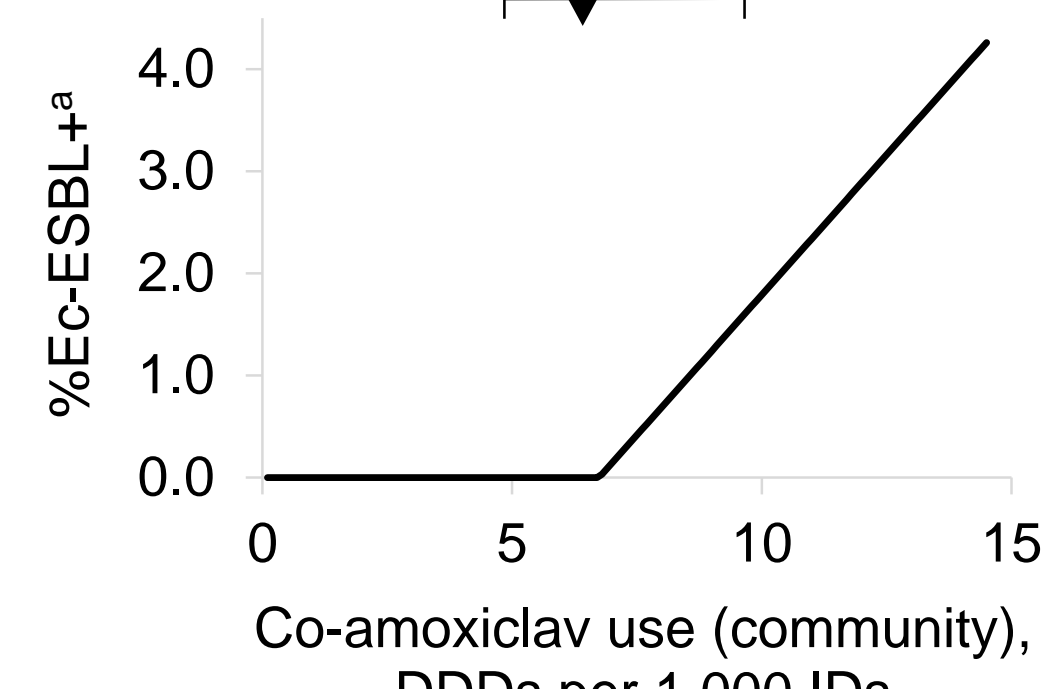
DDDs per 1,000 IDs 

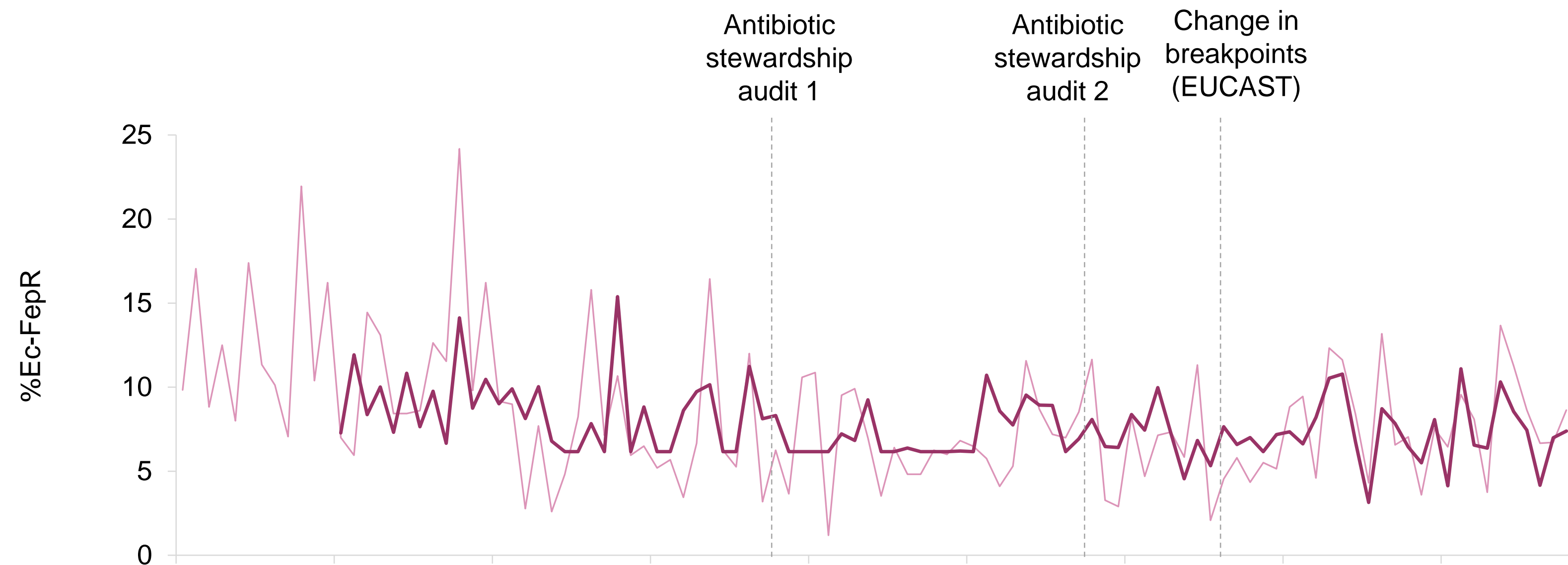

\section{— Observed \%Ec-FepR}

—Non-linear TSA model

b

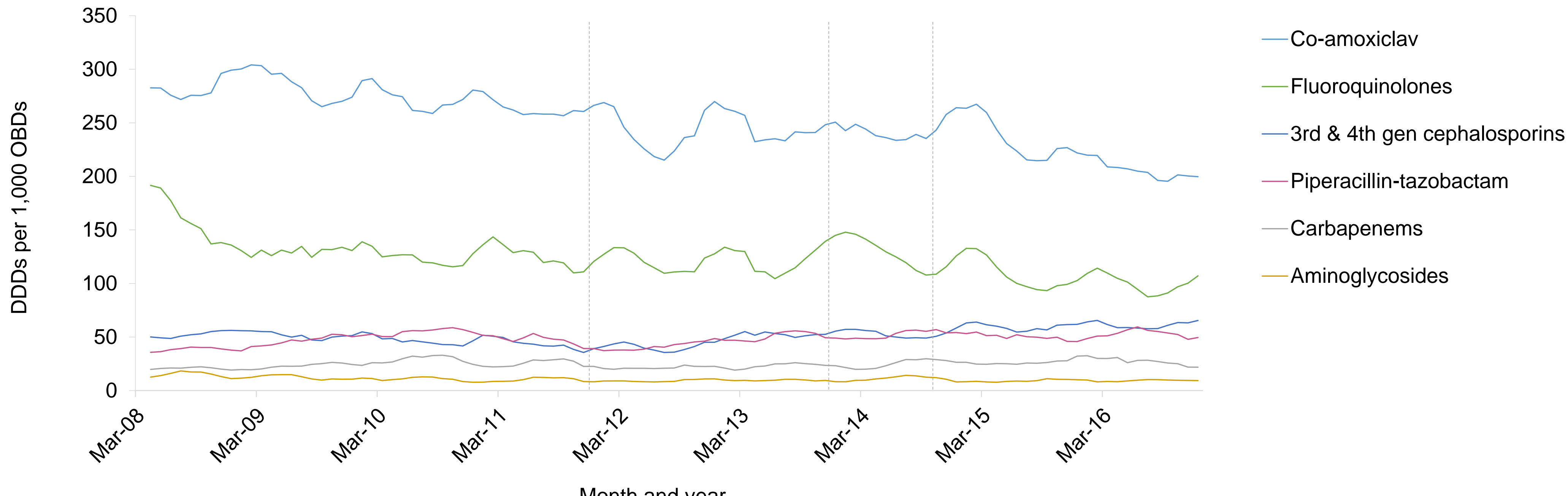

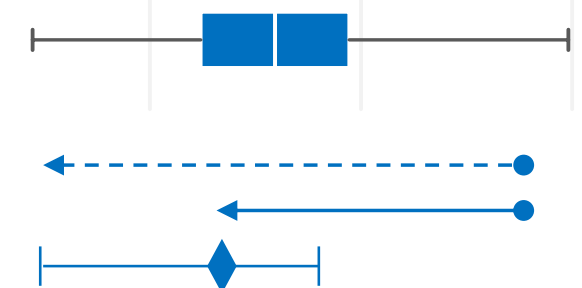

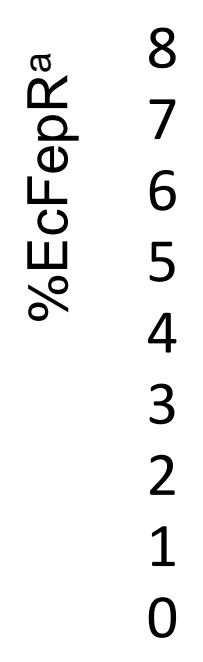

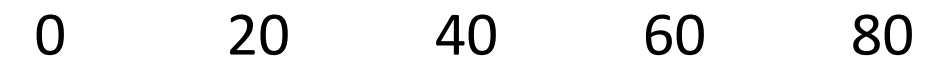

3rd \& 4th gen cephalosporin use, DDDs per 1,000 OBDs
Month and year

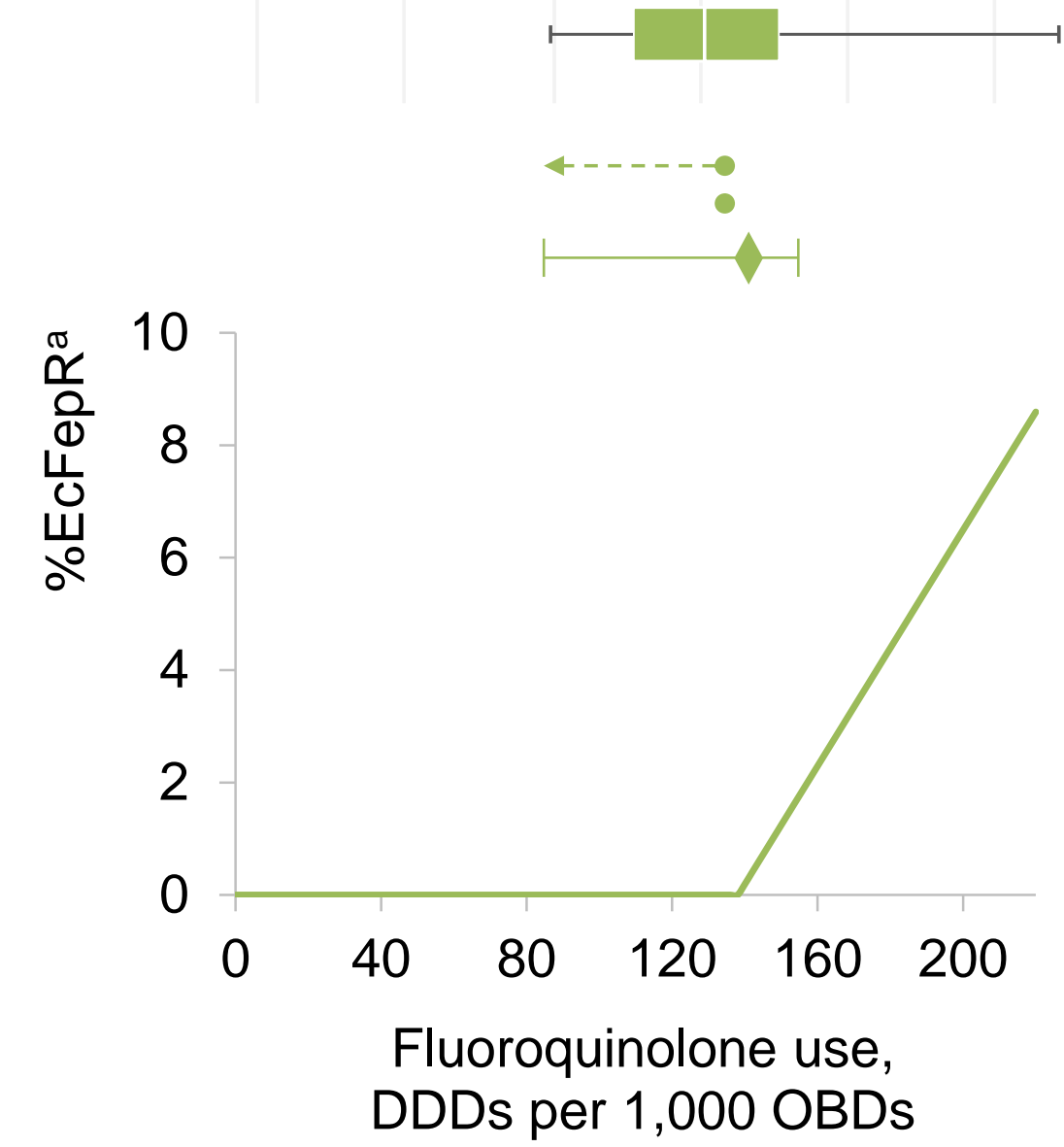

Median (IQR) and range of monthly antibiotic use

Estimated change in outcome by level of explanatory variable

Threshold and $95 \% \mathrm{Cl}$

Current use level (circle) and suggested change in use for standard (solid arrow) or conservative (dashed arrow) policy options 
a

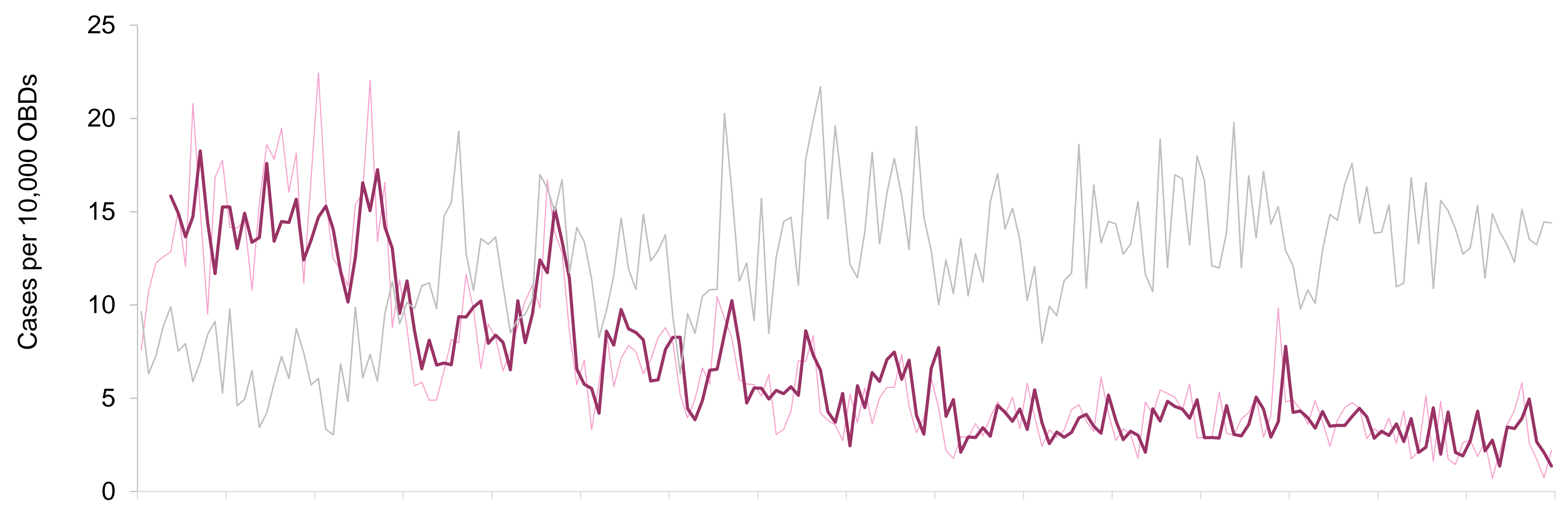

b
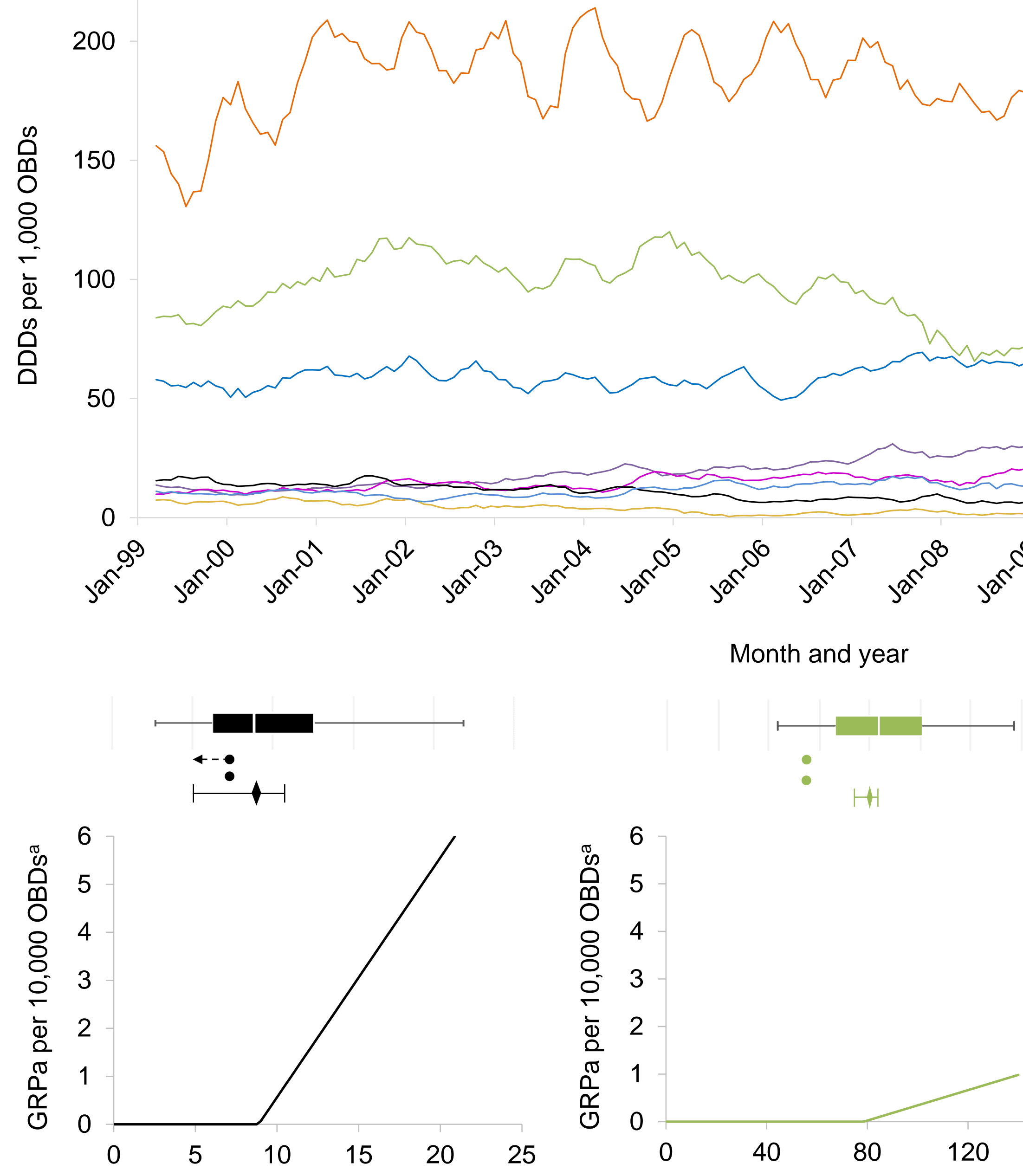

Tobramycin and Gentamicin use, DDDs per 1,000 OBDs

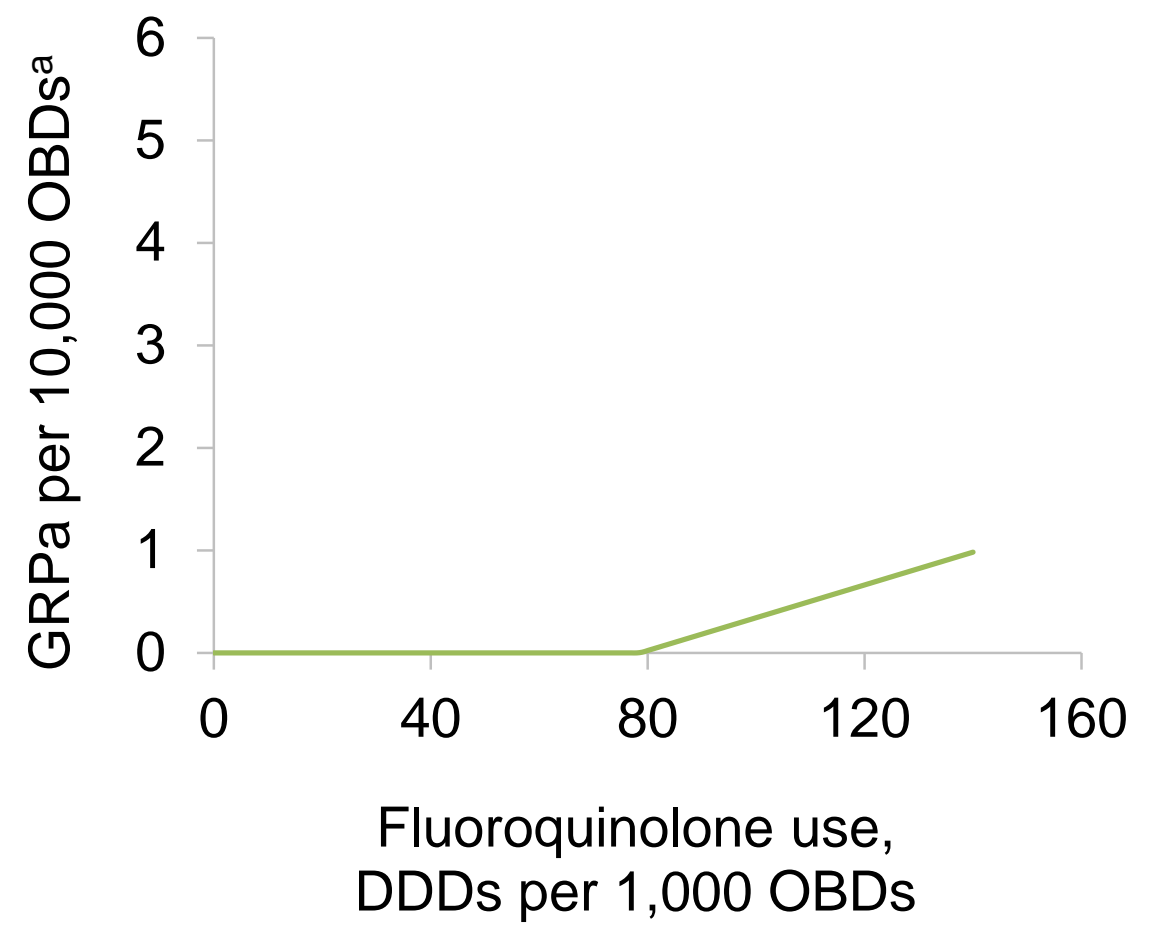

Median (IQR) and range of monthly antibiotic use

Estimated change in outcome by level of explanatory variable

Threshold and 95\% Cl

Current use level (circle) and suggested change in use for standard (solid arrow) or conservative (dashed arrow) policy options 


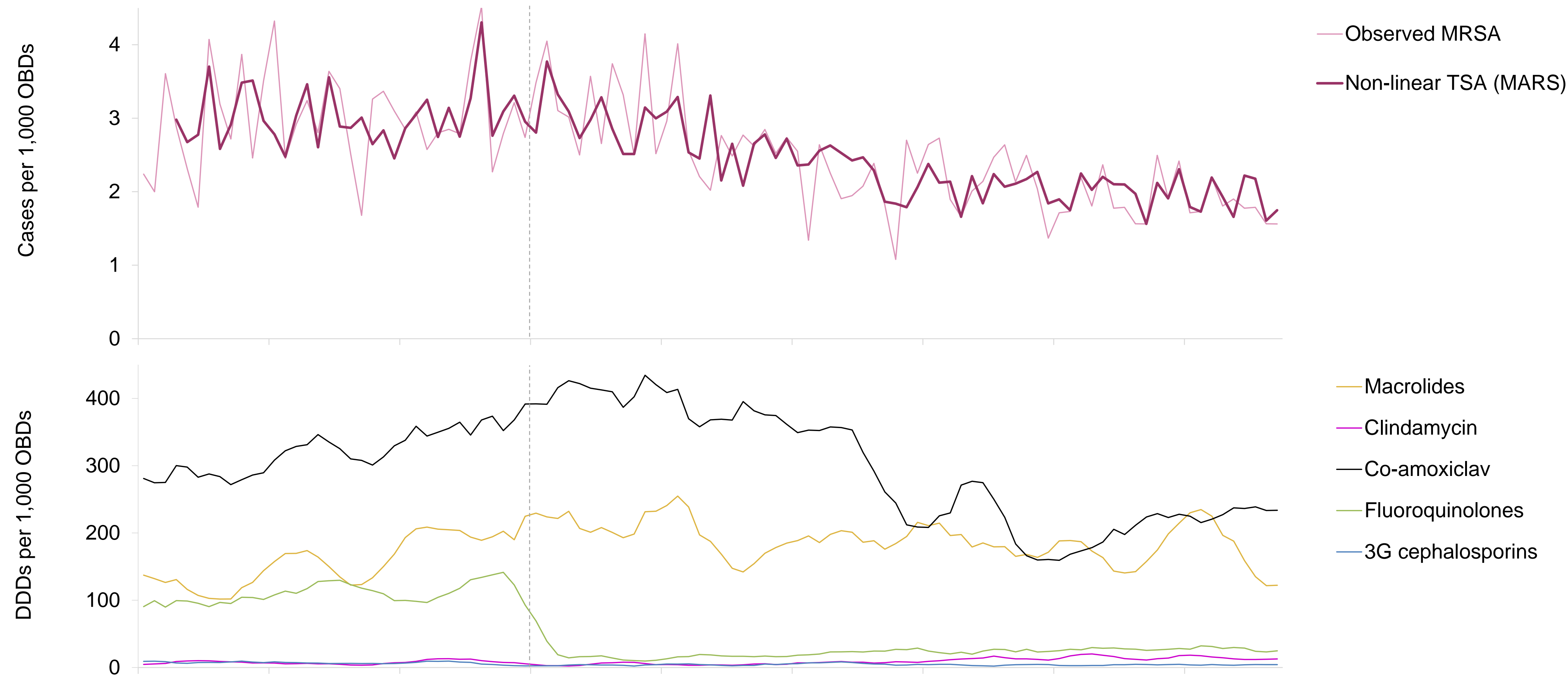

c

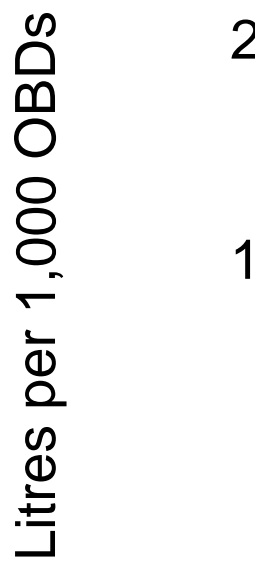

20

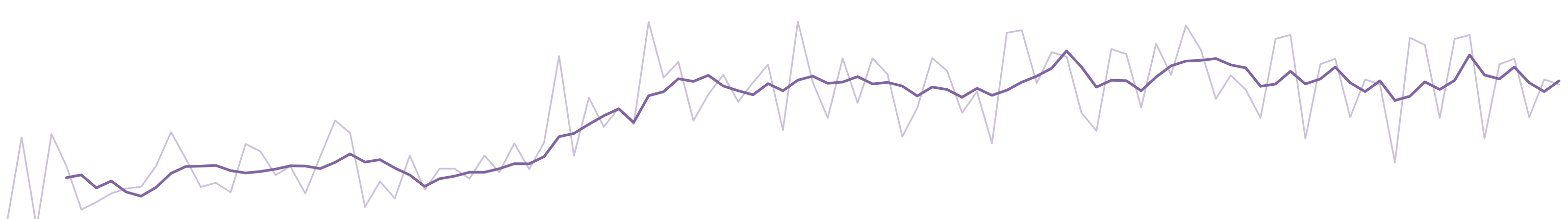

- ABHR use

_ $5 \mathrm{~m}$ moving average

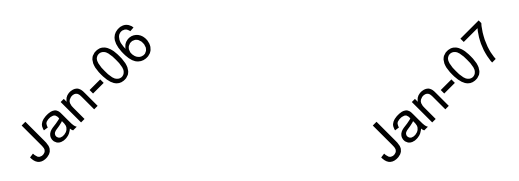

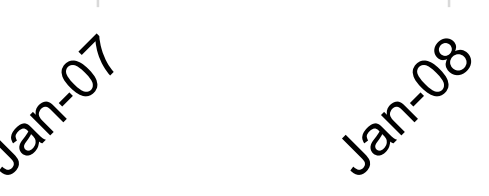<smiles>[CH][Co]</smiles> \\ Median (IQR) and range of monthly antibiotic use \\ Estimated change in outcome by level of explanatory variable}
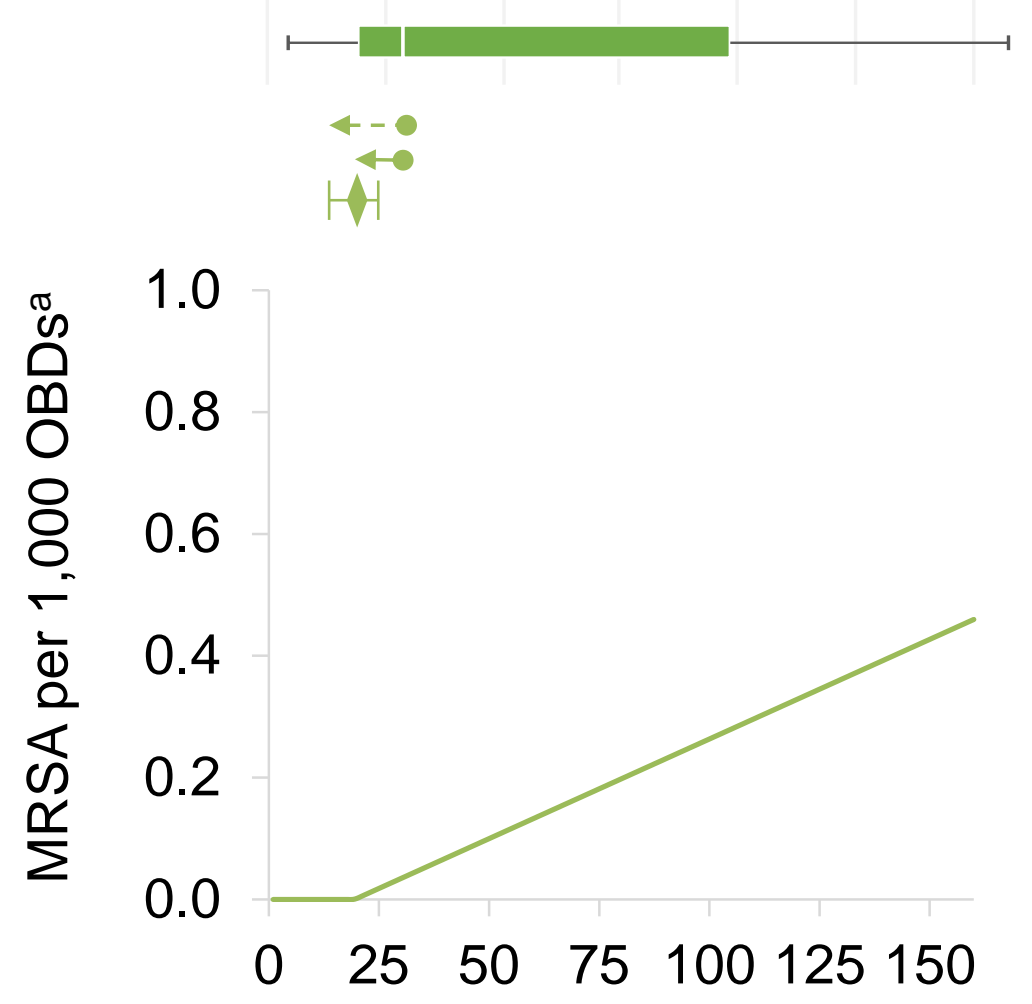

Fluoroquinolone use DDDs per 1,000 OBDs
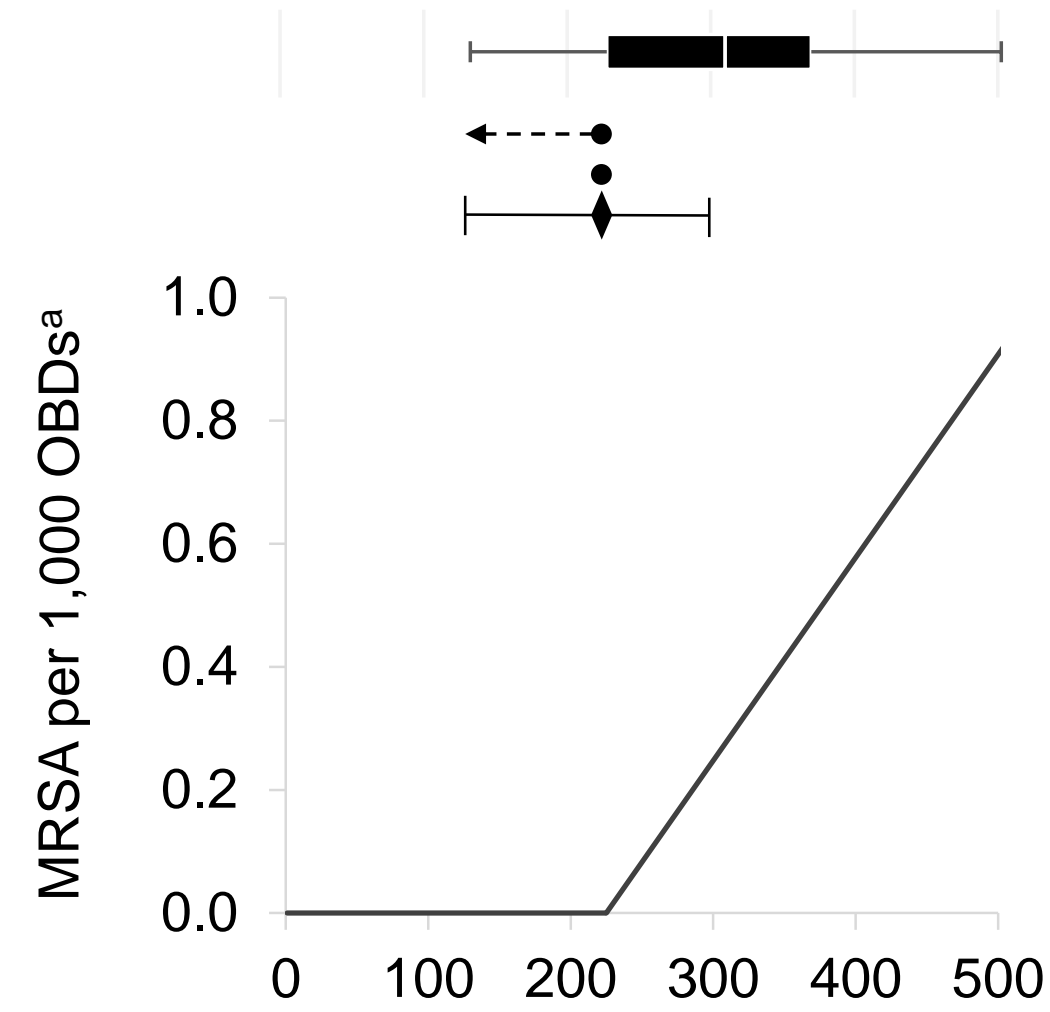

Co-amoxiclav use, DDDs per 1,000 OBDs
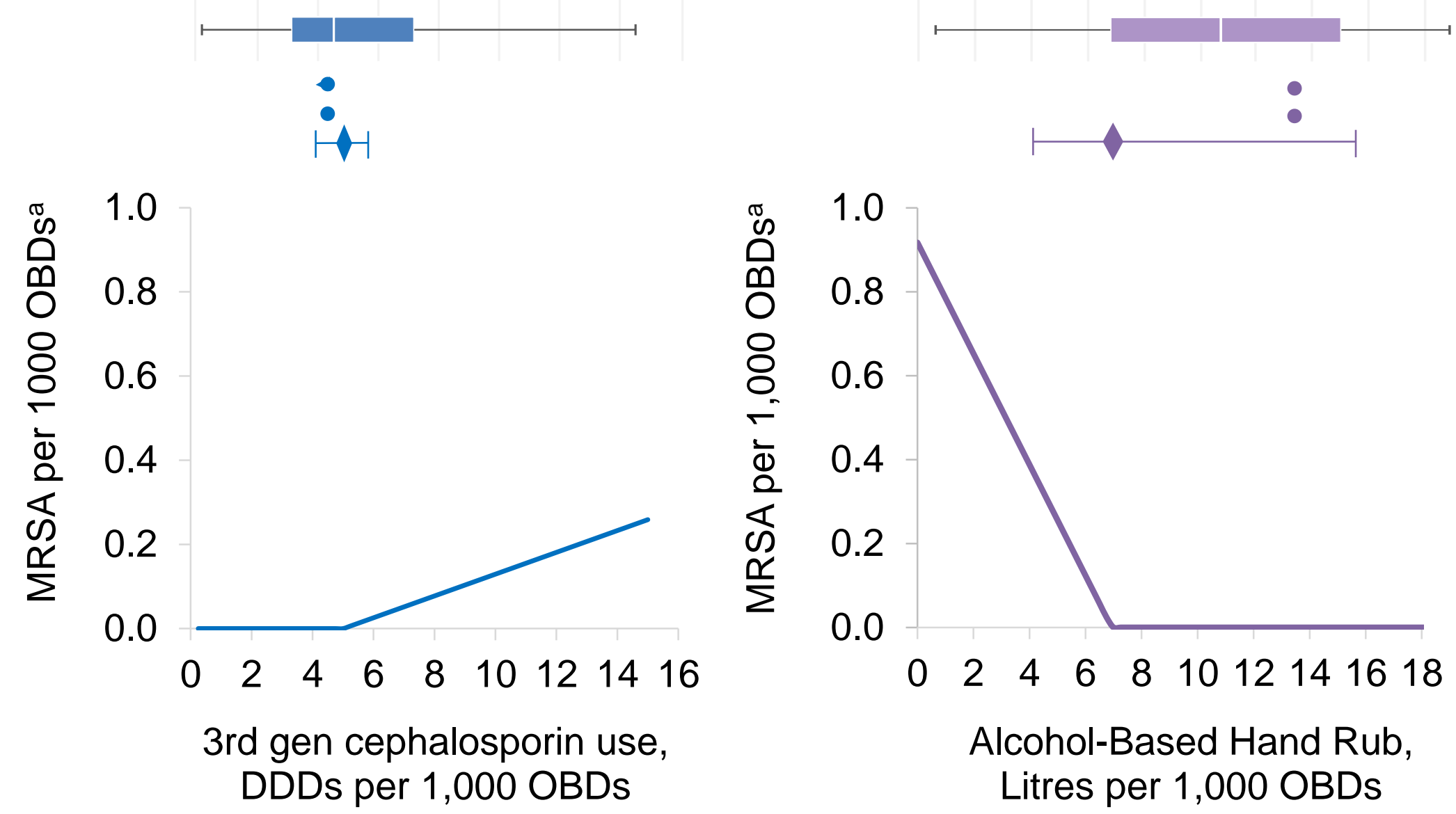